\title{
Cardiac Involvement in COVID-19 Patients: A Contemporary Review
}

\author{
Domenico Maria Carretta ${ }^{1}$, Aline Maria Silva ${ }^{2}$, Donato D'Agostino ${ }^{2}$, Skender Topi ${ }^{3}$, Roberto Lovero ${ }^{4}$, \\ Ioannis Alexandros Charitos ${ }^{5, *}$, Angelika Elzbieta Wegierska ${ }^{6}$, Monica Montagnani ${ }^{7,+(D)}$ and \\ Luigi Santacroce ${ }^{6, *}+$ (1D)
}

1 AOU Policlinico Consorziale di Bari-Ospedale Giovanni XXIII, Coronary Unit and Electrophysiology/Pacing Unit, Cardio-Thoracic Department, Policlinico University Hospital of Bari, 70124 Bari, Italy; carrettacardiologia@gmail.com

2 AOU Policlinico Consorziale di Bari-Ospedale Giovanni XXIII, Cardiac Surgery, Policlinico University Hospital of Bari, 70124 Bari, Italy; dr.alinemariasilva@gmail.com (A.M.S.); prof_d.dagostino@libero.it (D.D.)

3 Department of Clinical Disciplines, School of Technical Medical Sciences, University of Elbasan "A. Xhuvani", 3001 Elbasan, Albania; skender.topi@uniel.edu.al

4 AOU Policlinico Consorziale di Bari-Ospedale Giovanni XXIII, Clinical Pathology Unit, Policlinico University Hospital of Bari, 70124 Bari, Italy; robertolovero69@gmail.com

5 Emergency/Urgent Department, National Poisoning Center, Riuniti University Hospital of Foggia, 71122 Foggia, Italy

6 Department of Interdisciplinary Medicine, Microbiology and Virology Unit, University of Bari "Aldo Moro", Piazza G. Cesare, 11, 70124 Bari, Italy; angelika.wegierska@yahoo.it

7 Department of Biomedical Sciences and Human Oncology-Section of Pharmacology, School of Medicine, University of Bari "Aldo Moro", Policlinico University Hospital of Bari, p.zza G. Cesare 11, 70124 Bari, Italy; monica.montagnani@uniba.it

* Correspondence: alexanestesia@hotmail.com (I.A.C.); luigi.santacroce@uniba.it (L.S.)

check for

updates

Citation: Carretta, D.M.; Silva, A.M.; D'Agostino, D.; Topi, S.; Lovero, R.; Charitos, I.A.; Wegierska, A.E.; Montagnani, M.; Santacroce, L. Cardiac Involvement in COVID-19 Patients: A Contemporary Review. Infect. Dis. Rep. 2021, 13, 494-517. https://doi.org/10.3390/idr13020048

Academic Editor: Nicola Petrosillo

Received: 2 May 2021

Accepted: 26 May 2021

Published: 1 June 2021

Publisher's Note: MDPI stays neutral with regard to jurisdictional claims in published maps and institutional affiliations.

Copyright: (c) 2021 by the authors. Licensee MDPI, Basel, Switzerland. This article is an open access article distributed under the terms and conditions of the Creative Commons Attribution (CC BY) license (https:// creativecommons.org/licenses/by/ $4.0 /)$.
+ These authors equally contributed as co-last authors.

Abstract: Background: The widely variable clinical manifestations of SARS-CoV2 disease (COVID-19) range from asymptomatic infections to multiple organ failure and death. Among the organs affected is the heart. This does not only affect people who already have previous cardiovascular problems, but also healthy people. This is a reason not to overlook any symptoms or to perform targeted examinations, even if apparently unrelated to the heart, for quick recognition and timely therapy. Aim of the study: This review recapitulates the current state of knowledge on the potential mechanisms and manifestation of myocarditis in patients with COVID-19 infection. Methods: A web-based search of published data was performed for all relevant studies on patients diagnosed with a COVID-19induced acute myocarditis, and a total of 50 reports were included. The analysis of the studies evaluated highlights a male predominance, with the average age of patients being 55 years. The most common presenting symptoms included fever, shortness of breath, cough, and chest pain. Among ECG changes, non-specific ST-segment and T-wave amplitude alterations and ventricular tachycardia episodes were reported. Finally, we wanted to use a general evaluation without distinguishing between various countries, taking into consideration only the peer or reviewer, regardless of the declared value of the journals that have been published. Results and critical findings: The most common presenting symptoms included fever, shortness of breath, cough, and chest pain. Among ECG changes, non-specific ST-segment and T-wave amplitude alterations and ventricular tachycardia episodes were reported. In most patients, elevated levels of cardiac and inflammatory biomarkers were measured. Left ventricular dysfunction and hypokinesis were commonly exhibited symptoms. Cardiac Magnetic Resonance Imaging (CMRI) confirmed the diagnosis of myocarditis with features of cardiac edema and cardiac injury. Nine patients underwent histopathological examination. Treatment with corticosteroids and immunoglobulins was the most applied strategy following the administration of antivirals. Discussion: Despite the exponentially growing knowledge on the management of COVID-19 infection, current available data on SARS-CoV2-correlated myocarditis are still limited, and several difficulties may be encountered in the differential diagnosis of acute myocarditis in the context of COVID-19 disease. Conclusions: While diagnostic criteria and evaluation strategies 
for myocarditis are well described, no guidelines for the diagnosis and treatment of myocarditis in COVID-19 patients have yet been established. Therefore, further research is needed to advance the understanding of this disease process and define the most appropriate strategic approach in these patients.

Keywords: SARS-CoV-2; viral myocarditis; cardiomyopathy; endomyocardial biopsy; cardiac magnetic resonance; cardiovascular therapy

\section{Introduction}

Severe acute respiratory syndrome coronavirus 2 (SARS-CoV-2) is a new variant form of coronavirus identified as the pathogen responsible for coronavirus disease 2019 (COVID-19). SARS-CoV-2 is like the severe acute respiratory syndrome coronavirus (SARS-CoV) and the Middle East respiratory syndrome coronavirus (MERS-CoV), and is believed to be the result of zoonotic transmission to humans from bats [1-3].

In December 2019, a COVID-19 outbreak was first reported in Wuhan, China, and in March 2020, the World Health Organization (WHO) declared a COVID-19 global pandemic after confirming cases on five continents [3,4].

Millions of COVID-19 cases have been reported so far, and efforts are underway to better define the epidemiology of the disease. Clinical manifestations of COVID-19 are widely variable and range from asymptomatic infection to multi-organ failure and death [4-6].

Although pulmonary involvement is the most common and most virulent clinical manifestation, cardiovascular symptoms have been found in $30 \%$ of hospitalized patients, sometimes associated with worse outcomes [6-8]. The understanding of the pathophysiology for the occurrence of myocarditis in COVID-19 is currently limited to clinical case reports. This review recapitulates the current knowledge on the potential mechanisms and manifestations of heart damage, and specifically of myocarditis, in patients with COVID-19 infection, in the attempt to clarify whether they were caused by direct viral damage or were secondary to inadequate host immune response. Here, clinical reports of myocarditis in COVID-19 patients are summarized and described to help shed light on this field, and increase our understanding of the clinical features, diagnostic process, and outcomes of this rare but often serious disease complication [9-16].

\section{Methods}

A web-based search of the published medical literature was performed for all relevant studies on patients diagnosed with COVID-19-induced acute myocarditis using the LitCovid, PubMed, Cochrane Library, Embase databases, Google Scholar, Elsevier Connect, and Web of Science, using the following keywords: "COVID-19", "coronavirus", and "SARS-CoV-2", in combination with the term "myocarditis". The identified reports were independently screened by two investigators (AMS and IAC). Only papers written in English were included. Each paper was retrieved, and its references were reviewed to identify additional studies. For each case, age, gender, clinical presentation, laboratory results, electrocardiogram, echocardiography, cardiac imaging, and endomyocardial biopsy data were considered. Only articles reporting myocarditis or myocardial infection in association with COVID-19 were included. Articles were initially selected based on whether the titles or abstracts met the inclusion criteria. Full-text articles that did not meet the appropriate inclusion criteria were excluded. Data extracted from articles include first author name, study design, and study sample.

\section{Results and Critical Findings}

For this review, 1296 articles were initially found by searching the indicated databases with the above-mentioned key words, and 864 studies underwent further evaluation after 
the removal of duplicates. Among the 65 articles meeting the inclusion criteria were 15 reviews, 36 case reports, 3 case series, 2 cohort studies, 5 retrospective studies, 2 metaanalyses, 1 single-center study, and 1 prospective study. All articles were published in 2020 . Studies were carried out in Asia, Europe, and America. Tables 1 and 2 below summarize the results from the published studies.

Table 1. Characteristics of published cases of SARS-CoV-2-induced myocarditis.

\begin{tabular}{|c|c|c|c|c|c|c|}
\hline Authors & Study Design & $\begin{array}{l}\text { Sample of } \\
\text { Study }\end{array}$ & $\begin{array}{l}\text { Presenting } \\
\text { Symptoms }\end{array}$ & $\begin{array}{c}\text { Cardiac } \\
\text { Biomarker }\end{array}$ & Results & Treatment \\
\hline $\begin{array}{l}\text { Asif and Ali } \\
\text { [17] }\end{array}$ & Case report & $\begin{array}{l}\text { 64-year-old } \\
\text { man }\end{array}$ & Dyspnea & $\begin{array}{l}\text { Troponin: } \\
0.17 \mathrm{ng} / \mathrm{mL} \\
\text { NT-proBNP: - }\end{array}$ & $\begin{array}{l}\text { ECG: T } \\
\text { Echo: - } \\
\text { CMR: NR } \\
\text { EMB/PM:NR }\end{array}$ & $\begin{array}{l}\text { Aspirin, clopidogrel, } \\
\text { heparin, azithromycin, } \\
\text { hydroxychloroquine, } \\
\text { meropenem, propofol, } \\
\text { fentanyl, atracurium, } \\
\text { vasopressin, } \\
\text { tocilizumab }\end{array}$ \\
\hline $\begin{array}{l}\text { Asif and Ali } \\
\text { [17] }\end{array}$ & Case report & $\begin{array}{l}\text { 71-year-old } \\
\text { man }\end{array}$ & $\begin{array}{l}\text { Fever, cough, and } \\
\text { dyspnea }\end{array}$ & $\begin{array}{l}\text { Troponin: } \\
1.6 \mathrm{ng} / \mathrm{mL} \\
\text { NT-proBNP: - }\end{array}$ & $\begin{array}{l}\text { ECG: ST, Q } \\
\text { Echo: - } \\
\text { CMR: NR } \\
\text { EMB/PM:NR }\end{array}$ & $\begin{array}{l}\text { Aspirin, clopidogrel, } \\
\text { heparin, azithromycin, } \\
\text { cefepime, vancomycin, } \\
\text { tocilizumab, fentanyl, } \\
\text { midazolam }\end{array}$ \\
\hline Beri et al. [18] & Case report & $\begin{array}{l}\text { 72-year old } \\
\text { man }\end{array}$ & $\begin{array}{l}\text { Chest tightness, } \\
\text { shortness of } \\
\text { breath, cough, } \\
\text { fatigue, and } \\
\text { intermittent } \\
\text { diarrhea }\end{array}$ & $\begin{array}{l}\text { Troponin: } \\
\text { - } \\
\text { NT-proBNP: - }\end{array}$ & $\begin{array}{l}\text { ECG: ST, TV } \\
\text { Echo: ventricular } \\
\text { dysfunction, } \\
\text { cardiomegaly } \\
\text { CMR: NR } \\
\text { EMB/PM: NR }\end{array}$ & NR \\
\hline $\begin{array}{l}\text { Bernal-Torres } \\
\text { et al. [19] }\end{array}$ & Case report & $\begin{array}{l}\text { 38-year-old } \\
\text { woman }\end{array}$ & $\begin{array}{l}\text { Palpitations, } \\
\text { general malaise }\end{array}$ & $\begin{array}{l}\text { Troponin: } \\
1190 \mathrm{ng} / \mathrm{mL} \\
\text { NT-proBNP: } \\
13,000 \mathrm{pg} / \mathrm{mL}\end{array}$ & $\begin{array}{l}\text { ECG: ST } \\
\text { Echo: LVEF 30\%, } \\
\text { hypokinesia } \\
\text { CMR: Edema+ } \\
\text { Injury+ } \\
\text { EMB/PM: NR }\end{array}$ & $\begin{array}{l}\text { Methylprednisolone, } \\
\text { immunoglobulin, } \\
\text { hydroxychloroquine, } \\
\text { azithromycin, } \\
\text { lopinavir/ritonavir }\end{array}$ \\
\hline $\begin{array}{l}\text { Beşler and } \\
\text { Arslan [20] }\end{array}$ & Case report & $\begin{array}{l}\text { 20-year-old } \\
\text { man }\end{array}$ & $\begin{array}{l}\text { Febrile sensation } \\
\text { and chest pain }\end{array}$ & $\begin{array}{l}\text { Troponin: } \\
0.572 \mathrm{ng} / \mathrm{mL} \\
\text { NT-proBNP: } 127 \\
\text { pg/L }\end{array}$ & $\begin{array}{l}\text { ECG: - } \\
\text { Echo: - } \\
\text { CMR: Edema+ } \\
\text { Injury+ } \\
\text { EMB/PM:NR }\end{array}$ & $\begin{array}{l}\text { Hydroxychloroquine, } \\
\text { azithromycin, } \\
\text { ceftriaxone, tigecycline, } \\
\text { favipiravir, colchicine }\end{array}$ \\
\hline $\begin{array}{l}\text { Cizgici et al. } \\
{[21]}\end{array}$ & Case report & $\begin{array}{l}\text { 78-year-old } \\
\text { man }\end{array}$ & $\begin{array}{l}\text { Chest pain and } \\
\text { shortness of } \\
\text { breath. }\end{array}$ & $\begin{array}{l}\text { Troponin: } \\
\text { 998.1 ng/mL } \\
\text { NT-proBNP: } 127 \\
\text { pg/mL }\end{array}$ & $\begin{array}{l}\text { ECG: FA, ST } \\
\text { Echo: - } \\
\text { CMR: Edema+ } \\
\text { Injury+ } \\
\text { EMB/PM:NR }\end{array}$ & $\begin{array}{l}\text { Furosemide, ACE-I, } \\
\text { beta-blocker, } \\
\text { COVID-19-specific } \\
\text { therapy }\end{array}$ \\
\hline Coyle et al. [22] & Case report & $\begin{array}{l}\text { 57-year-old } \\
\text { man }\end{array}$ & $\begin{array}{l}\text { Shortness of } \\
\text { breath, fevers, } \\
\text { cough, myalgia, } \\
\text { decreased } \\
\text { appetite, nausea, } \\
\text { and diarrhea }\end{array}$ & $\begin{array}{l}\text { Troponin: } \\
7.33 \mathrm{ng} / \mathrm{mL} \\
\text { NT-proBNP: } \\
1300 \mathrm{pg} / \mathrm{mL}\end{array}$ & $\begin{array}{l}\text { ECG: - } \\
\text { Echo: } \\
\text { Hypokinesia, } \\
\text { LVEF 35-40\% } \\
\text { CMR: Edema+ } \\
\text { Injury+ } \\
\text { EMB/PM:NR }\end{array}$ & $\begin{array}{l}\text { Hydroxychloroquine, } \\
\text { methylprednisolone, } \\
\text { azithromycin, } \\
\text { ceftriaxone, colchicine }\end{array}$ \\
\hline $\begin{array}{c}\text { Craver et al. } \\
\text { [23] }\end{array}$ & Case report & $\begin{array}{l}\text { 17-year-old } \\
\text { man }\end{array}$ & $\begin{array}{l}\text { Full cardiac arrest } \\
\text { after a 2-day } \\
\text { history of } \\
\text { headache, } \\
\text { dizziness, nausea, } \\
\text { and vomiting }\end{array}$ & NR & $\begin{array}{l}\text { ECG: NR } \\
\text { Echo: NR } \\
\text { CMR: NR } \\
\text { EMB/PM: } \\
\text { Immune cell } \\
\text { infiltrate, necrosis }\end{array}$ & NR \\
\hline
\end{tabular}


Table 1. Cont.

\begin{tabular}{|c|c|c|c|c|c|c|}
\hline Authors & Study Design & $\begin{array}{l}\text { Sample of } \\
\text { Study }\end{array}$ & $\begin{array}{l}\text { Presenting } \\
\text { Symptoms }\end{array}$ & $\begin{array}{c}\text { Cardiac } \\
\text { Biomarker }\end{array}$ & Results & Treatment \\
\hline $\begin{array}{c}\text { Dolhnikoff et al. } \\
\text { [24] }\end{array}$ & Case report & $\begin{array}{l}\text { 11-year-old } \\
\text { boy }\end{array}$ & $\begin{array}{l}\text { Fever, } \\
\text { odynophagia, } \\
\text { myalgia, and } \\
\text { abdominal pain }\end{array}$ & $\begin{array}{l}\text { Troponin: } \\
0.342 \text { ng/mL } \\
\text { NT-proBNP: - }\end{array}$ & $\begin{array}{l}\text { ECG: TV } \\
\text { Echo: LVEF 31\%, } \\
\text { hypokinesia } \\
\text { CMR: Edema+ } \\
\text { Injury+ } \\
\text { EMB/PM: viral } \\
\text { particles in different } \\
\text { cell lineages of the } \\
\text { heart, including } \\
\text { cardiomyocytes, } \\
\text { endothelial cells, } \\
\text { mesenchymal cells }\end{array}$ & $\begin{array}{l}\text { Epinephrine, } \\
\text { furosemide, ceftriaxone, } \\
\text { azithromycin }\end{array}$ \\
\hline $\begin{array}{l}\text { Doyen et al. } \\
\text { [25] }\end{array}$ & Case report & $\begin{array}{l}\text { 69-year-old } \\
\text { man }\end{array}$ & $\begin{array}{l}\text { Cough, fever, } \\
\text { dyspnea }\end{array}$ & $\begin{array}{l}\text { Troponin: } \\
9002 \mathrm{ng} / \mathrm{mL} \\
\text { NT-proBNP: } \\
22.600 \mathrm{pg} / \mathrm{mL}\end{array}$ & $\begin{array}{l}\text { ECG: T } \\
\text { Echo: LVH } \\
\text { CMR: Edema+ } \\
\text { Injury+ } \\
\text { EMB/PM: NR }\end{array}$ & $\begin{array}{l}\text { Hydrocortisone, } \\
\text { aspirin, fondaparinux }\end{array}$ \\
\hline Fried et al. [26] & Case report & $\begin{array}{l}\text { 64-year-old } \\
\text { woman }\end{array}$ & Chest pressure & $\begin{array}{l}\text { Troponin: } \\
\text { 18,6 ng/mL } \\
\text { NT-proBNP: - }\end{array}$ & $\begin{array}{l}\text { ECG: Sinusal tachy, } \\
\text { ST } \\
\text { Echo: LVEF 30\%, } \\
\text { pericardial effusion } \\
\text { CMR: NR } \\
\text { EMB/PM: NR }\end{array}$ & $\begin{array}{l}\text { Hydroxychloroquine, } \\
\text { dobutamine, IABP }\end{array}$ \\
\hline Hu et al. [27] & Case report & $\begin{array}{l}\text { 37-year-old } \\
\text { man }\end{array}$ & $\begin{array}{l}\text { Chest pain, } \\
\text { dyspnea, and } \\
\text { diarrhea }\end{array}$ & $\begin{array}{l}\text { Troponin: } \\
\text { 10,000 ng/mL } \\
\text { NT-proBNP: } 2102 \\
\text { pg/mL }\end{array}$ & $\begin{array}{l}\text { ECG: ST } \\
\text { Echo: LVD, LVEF } \\
27 \% \\
\text { CMR: NR } \\
\text { EMB/PM:NR }\end{array}$ & $\begin{array}{l}\text { Methylprednisolone, } \\
\text { immunoglobulin, } \\
\text { norepinephrine, } \\
\text { diuretic, milrinone, } \\
\text { pantoprazole, } \\
\text { piperacillin/sulbactam }\end{array}$ \\
\hline $\begin{array}{c}\text { Inciardi et al. } \\
\text { [28] }\end{array}$ & Case report & $\begin{array}{l}\text { 53-year-old } \\
\text { woman }\end{array}$ & Fever and cough & $\begin{array}{l}\text { Troponin: } \\
0,24 \text { ng/mL } \\
\text { NT-proBNP: } 5647 \\
\text { pg/mL }\end{array}$ & $\begin{array}{l}\text { ECG: ST, T } \\
\text { Echo: Hypokinesia, } \\
\text { LVEF } 40 \% \text {, } \\
\text { pericardial effusion } \\
\text { CMR: Edema+ } \\
\text { Injury+ } \\
\text { EMB/PM: NR }\end{array}$ & $\begin{array}{l}\text { Hydroxychloroquine, } \\
\text { lopinavir/ritonavir, } \\
\text { methylprednisolone, } \\
\text { bisoprolol, kanrenone, } \\
\text { furosemide }\end{array}$ \\
\hline $\begin{array}{l}\text { Irabien- Ortiz } \\
\text { et al. [29] }\end{array}$ & Case report & $\begin{array}{l}\text { 59-year-old } \\
\text { woman }\end{array}$ & $\begin{array}{l}\text { Anginal chest } \\
\text { pain in the } \\
\text { absence of } \\
\text { respiratory } \\
\text { symptoms }\end{array}$ & $\begin{array}{l}\text { Troponin: } \\
\text { 1100 ng/mL } \\
\text { NT-proBNP: } 4421 \\
\text { pg/mL }\end{array}$ & $\begin{array}{l}\text { ECG: ST } \\
\text { Echo: LVH } \\
\text { CMR: Edema+ } \\
\text { Injury+ } \\
\text { EMB/PM:NR }\end{array}$ & $\begin{array}{l}\text { Immunoglobulins, } \\
\text { methylprednisolone, } \\
\text { ritonavir/lopinavir, } \\
\text { interferon-B }\end{array}$ \\
\hline Jacobs et al. [30] & Case report & $\begin{array}{l}\text { 48-year-old } \\
\text { man }\end{array}$ & $\begin{array}{l}\text { Fever, diarrhoea, } \\
\text { cough, dysosmia, } \\
\text { and dyspnea }\end{array}$ & $\begin{array}{l}\text { Troponin: } 14 \\
932 \text { ng/mL } \\
\text { NT-proBNP: } 9223 \\
\text { pg/mL }\end{array}$ & $\begin{array}{l}\text { ECG: T } \\
\text { Echo: LVH, } \\
\text { hyperdynamic } \\
\text { ventricular function } \\
\text { CMR: NR } \\
\text { EMB/PM: Immune } \\
\text { cell infiltrate, } \\
\text { edema, necrosis }\end{array}$ & $\begin{array}{l}\text { Hydroxychloroquine, } \\
\text { azithromycin, ECMO }\end{array}$ \\
\hline $\begin{array}{c}\text { Juusela et al. } \\
\text { [31] }\end{array}$ & Case report & $\begin{array}{l}\text { 45-year-old } \\
\text { woman, } \\
\text { pregnant } \\
\text { (39 weeks) }\end{array}$ & $\begin{array}{l}\text { Contractions and } \\
\text { emesis }\end{array}$ & $\begin{array}{l}\text { Troponin: } \\
0.046 \text { ng/mL } \\
\text { NT-proBNP: } 114 \\
\text { pg/mL }\end{array}$ & $\begin{array}{l}\text { ECG: - } \\
\text { Echo: LVEF 40\% } \\
\text { CMR: NR } \\
\text { EMB/PM:NR }\end{array}$ & $\begin{array}{l}\text { Methylprednisolone, } \\
\text { hydroxychloroquine, } \\
\text { tocilizumab, cesarean }\end{array}$ \\
\hline $\begin{array}{c}\text { Juusela et al. } \\
\text { [31] }\end{array}$ & Case report & $\begin{array}{l}\text { 26-year-old } \\
\text { woman, } \\
\text { pregnant } \\
\text { (33 weeks) }\end{array}$ & $\begin{array}{l}\text { Shortness of } \\
\text { breath, dyspnea, }\end{array}$ & $\begin{array}{l}\text { Troponin: } \\
0.046 \text { ng/mL } \\
\text { NT-proBNP: }<10 \\
\text { pg/mL }\end{array}$ & $\begin{array}{l}\text { ECG: VT } \\
\text { Echo: Hypokinesia, } \\
\text { LVEF } 40 \% \\
\text { CMR: - } \\
\text { EMB/PM:NR }\end{array}$ & Metoprolol, cesarean \\
\hline
\end{tabular}


Table 1. Cont.

\begin{tabular}{|c|c|c|c|c|c|c|}
\hline Authors & Study Design & $\begin{array}{l}\text { Sample of } \\
\text { Study }\end{array}$ & $\begin{array}{l}\text { Presenting } \\
\text { Symptoms }\end{array}$ & $\begin{array}{c}\text { Cardiac } \\
\text { Biomarker }\end{array}$ & Results & Treatment \\
\hline Kim et al. [32] & Case report & $\begin{array}{l}\text { 21-year-old } \\
\text { woman }\end{array}$ & $\begin{array}{l}\text { Coughing, } \\
\text { sputum, diarrhea, } \\
\text { and shortness of } \\
\text { breath }\end{array}$ & $\begin{array}{l}\text { Troponin: } \\
\text { 1.26 ng/mL } \\
\text { NT-proBNP: } 1929 \\
\text { pg/mL }\end{array}$ & $\begin{array}{l}\text { ECG: VE } \\
\text { Echo: Severe LV } \\
\text { systolic dysfunction } \\
\text { CMR: Edema+ } \\
\text { Injury+ } \\
\text { EMB/PM: NR }\end{array}$ & NR \\
\hline $\begin{array}{c}\text { Luetkens et al. } \\
\text { [33] }\end{array}$ & Case report & $\begin{array}{l}\text { 79-year-old } \\
\text { man }\end{array}$ & $\begin{array}{l}\text { Fatigue, shortness } \\
\text { of breath, } \\
\text { recurrent } \\
\text { syncopes }\end{array}$ & $\begin{array}{l}\text { Troponin: } \\
\text { 63,5 ng/mL } \\
\text { NT-proBNP: } 1178 \\
\text { pg/mL }\end{array}$ & $\begin{array}{l}\text { ECG: - } \\
\text { Echo: Hypokinesia, } \\
\text { LVEF } 49 \% \\
\text { CMR: Edema+ } \\
\text { Injury+ } \\
\text { EMB/PM: NR }\end{array}$ & NR \\
\hline $\begin{array}{l}\text { Naneishvili } \\
\text { et al. [34] }\end{array}$ & Case report & $\begin{array}{l}\text { 44-year-old } \\
\text { woman }\end{array}$ & $\begin{array}{l}\text { Fever, lethargy, } \\
\text { muscle aches and } \\
\text { two episodes of } \\
\text { syncope }\end{array}$ & $\begin{array}{l}\text { Troponin: } \\
639 \text { ng/mL } \\
\text { NT-proBNP: - }\end{array}$ & $\begin{array}{l}\text { ECG: FA } \\
\text { Echo: LVEF 37\%, } \\
\text { LVH, pericardial } \\
\text { effusion } \\
\text { CMR: NR } \\
\text { EMB/PM: NR }\end{array}$ & $\begin{array}{l}\text { Methylprednisolone, } \\
\text { remdesvir, amiodarone, } \\
\text { milrinone, inotropic } \\
\text { support }\end{array}$ \\
\hline $\begin{array}{c}\text { Oberweis et al. } \\
{[35]}\end{array}$ & Case report & $\begin{array}{l}\text { 8-year-old } \\
\text { male }\end{array}$ & $\begin{array}{l}\text { Fever, coughing, } \\
\text { weight loss, and } \\
\text { severe fatigue }\end{array}$ & $\begin{array}{l}\text { Troponin: } \\
0.044 \text { ng/mL } \\
\text { NT-proBNP: } 5112 \\
\text { pg/mL }\end{array}$ & $\begin{array}{l}\text { ECG: ST } \\
\text { Echo: LVEF 21\%, } \\
\text { pericardial effusion } \\
\text { CMR: Edema+ } \\
\text { Injury+ } \\
\text { EMB/PM: NR }\end{array}$ & $\begin{array}{l}\text { Heparin, dobutamine, } \\
\text { immunoglobulins, } \\
\text { milrinone, tocilizumab }\end{array}$ \\
\hline $\begin{array}{c}\text { Oleszak et al. } \\
{[36]}\end{array}$ & Case report & $\begin{array}{l}\text { 52-year-old } \\
\text { man }\end{array}$ & $\begin{array}{l}\text { Cough, fever, } \\
\text { shortness of } \\
\text { breath, and a } \\
\text { single episode of } \\
\text { trace hemoptysis }\end{array}$ & $\begin{array}{l}\text { Troponin: } \\
0.017 \text { ng/mL } \\
\text { NT-proBNP: } 1220 \\
\text { pg/mL }\end{array}$ & $\begin{array}{l}\text { ECG: Sinusal tachy } \\
\text { Echo: LVEF 25\%, } \\
\text { LVH } \\
\text { CMR: NR } \\
\text { EMB/PM: NR }\end{array}$ & $\begin{array}{l}\text { Ceftriaxone, } \\
\text { azithromycin, } \\
\text { hydroxychloroquine, } \\
\text { furosemide }\end{array}$ \\
\hline Paul et al. [37] & Case report & $\begin{array}{l}\text { 35-year-old } \\
\text { man }\end{array}$ & $\begin{array}{l}\text { Chest pain and } \\
\text { fatigue }\end{array}$ & $\begin{array}{l}\text { Troponin: } \\
\text { 2885 ng/mL } \\
\text { NT-proBNP: - }\end{array}$ & $\begin{array}{l}\text { ECG: repolarization } \\
\text { changes } \\
\text { Echo: - } \\
\text { CMR: Edema+ } \\
\text { Injury+ } \\
\text { EMB/PM:NR }\end{array}$ & Ramipril, bisoprolol \\
\hline Pavon et al. [38] & Case report & $\begin{array}{l}\text { 64-year-old } \\
\text { man }\end{array}$ & $\begin{array}{l}\text { Chest pain and } \\
\text { dyspnea }\end{array}$ & $\begin{array}{l}\text { Troponin: } 1843 \\
\text { ng/L } \\
\text { NT-proBNP: - }\end{array}$ & $\begin{array}{l}\text { ECG: - } \\
\text { Echo: LVEF 40\% } \\
\text { CMR: Edema+ } \\
\text { Injury+ } \\
\text { EMB/PM: NR }\end{array}$ & Piperacillin/tazobactam \\
\hline $\begin{array}{l}\text { Radbel et al. } \\
\text { [39] }\end{array}$ & Case report & $\begin{array}{l}\text { 40-year-old } \\
\text { man }\end{array}$ & $\begin{array}{l}\text { Fever, dry cough, } \\
\text { and dyspnea }\end{array}$ & $\begin{array}{l}\text { Troponin: } \\
\text { 30,39 ng/mL } \\
\text { NT-proBNP: - }\end{array}$ & $\begin{array}{l}\text { ECG: ST } \\
\text { Echo: hypokinesia } \\
\text { CMR: NR } \\
\text { EMB/PM: NR }\end{array}$ & $\begin{array}{l}\text { Hydroxychloroquine, } \\
\text { azithromycin, } \\
\text { tocilizumab }\end{array}$ \\
\hline Rauf et al. [40] & Case report & 5-year-old boy & $\begin{array}{l}\text { Fever, abdominal } \\
\text { pain and loose } \\
\text { stools, bulbar } \\
\text { conjunctivitis, } \\
\text { and extremity } \\
\text { edema }\end{array}$ & $\begin{array}{l}\text { Troponin: } \\
29 \mathrm{ng} / \mathrm{mL} \\
\text { NT-proBNP: } 8000 \\
\text { pg/mL }\end{array}$ & $\begin{array}{l}\text { ECG: - } \\
\text { Echo: LVEF 35\%, } \\
\text { hypokinesia } \\
\text { CMR: NR } \\
\text { EMB/PM: NR }\end{array}$ & $\begin{array}{l}\text { Inotropic support, } \\
\text { immunoglobulins, } \\
\text { aspirin, steroids, } \\
\text { diuretics }\end{array}$ \\
\hline Sala et al. [41] & Case report & $\begin{array}{l}\text { 43-year-old } \\
\text { woman }\end{array}$ & $\begin{array}{l}\text { Chest pain and } \\
\text { dyspnea }\end{array}$ & $\begin{array}{l}\text { Troponin: } \\
\text { 106 ng/mL } \\
\text { NT-proBNP: } 512 \\
\text { pg/mL }\end{array}$ & $\begin{array}{l}\text { ECG: ST } \\
\text { Echo: Hypokinesia, } \\
\text { LVEF } 43 \% \\
\text { CMR: Edema+ } \\
\text { Injury+ } \\
\text { EMB/PM: Immune } \\
\text { cell infiltrate, } \\
\text { edema, necrosis }\end{array}$ & $\begin{array}{l}\text { Lopinavir/ritonavir, } \\
\text { hydroxychloroquine }\end{array}$ \\
\hline
\end{tabular}


Table 1. Cont.

\begin{tabular}{|c|c|c|c|c|c|c|}
\hline Authors & $\begin{array}{l}\text { Study } \\
\text { Design }\end{array}$ & $\begin{array}{l}\text { Sample of } \\
\text { Study }\end{array}$ & $\begin{array}{l}\text { Presenting } \\
\text { Symptoms }\end{array}$ & $\begin{array}{c}\text { Cardiac } \\
\text { Biomarker }\end{array}$ & Results & Treatment \\
\hline $\begin{array}{l}\text { Sardari et al. } \\
\text { [42] }\end{array}$ & Case report & $\begin{array}{l}\text { 31-year-old } \\
\text { man }\end{array}$ & $\begin{array}{l}\text { Dyspnea on } \\
\text { exertion and } \\
\text { low-grade fever }\end{array}$ & $\begin{array}{l}\text { Troponin: } \\
<0.03 \mathrm{ng} / \mathrm{mL} \\
\text { NT-proBNP: - }\end{array}$ & $\begin{array}{l}\text { ECG: - } \\
\text { Echo: Mild LV } \\
\text { systolic dysfunction } \\
\text { CMR: Edema+ } \\
\text { Injury+ } \\
\text { EMB/PM:NR }\end{array}$ & Bisoprolol, lisinopril \\
\hline $\begin{array}{c}\text { Tavazzi et al. } \\
\text { [43] }\end{array}$ & Case report & $\begin{array}{l}\text { 69-year-old } \\
\text { woman }\end{array}$ & $\begin{array}{l}\text { Dyspnea, cough, } \\
\text { and weakness }\end{array}$ & $\begin{array}{l}\text { Troponin: } \\
4332 \text { ng/mL } \\
\text { NT-proBNP: - }\end{array}$ & $\begin{array}{l}\text { ECG: - } \\
\text { Echo: LVD, LVEF } \\
27 \% \\
\text { CMR: NR } \\
\text { EMB/PM: viral } \\
\text { particles within the } \\
\text { interstitial cells of } \\
\text { the myocardium, } \\
\text { edema, necrosis }\end{array}$ & IABP, ECMO \\
\hline $\begin{array}{l}\text { Trogen et al. } \\
\text { [44] }\end{array}$ & Case report & $\begin{array}{l}\text { 17-year-old } \\
\text { man }\end{array}$ & $\begin{array}{l}\text { Fever and neck } \\
\text { pain, diarrhea }\end{array}$ & $\begin{array}{l}\text { Troponin: } \\
2.97 \text { ng/mL } \\
\text { NT-proBNP: } 2124 \\
\text { pg/mL }\end{array}$ & $\begin{array}{l}\text { ECG: T } \\
\text { Echo: LVEF 40\% } \\
\text { CMR: Edema+ } \\
\text { Injury+ } \\
\text { EMB/PM: NR }\end{array}$ & $\begin{array}{l}\text { Hydroxychloroquine, } \\
\text { piperacillin-tazobactam, } \\
\text { enoxaparin }\end{array}$ \\
\hline $\begin{array}{c}\text { Warchoł et al. } \\
\text { [45] }\end{array}$ & Case report & $\begin{array}{l}\text { 74-year-old } \\
\text { man }\end{array}$ & $\begin{array}{l}\text { Palpitations, } \\
\text { general malaise }\end{array}$ & $\begin{array}{l}\text { Troponin: } \\
102 \text { ng/mL } \\
\text { NT-proBNP: } 2451 \\
\text { pg/mL }\end{array}$ & $\begin{array}{l}\text { ECG: VT } \\
\text { Echo: LVEF 25\% } \\
\text { CMR: Edema - } \\
\text { Injury+ } \\
\text { EMB/PM: NR }\end{array}$ & Metoprolol, amiodarone \\
\hline Yuan et al. [46] & Case report & $\begin{array}{l}\text { 33-year-old } \\
\text { man }\end{array}$ & $\begin{array}{l}\text { Chest pain, fever, } \\
\text { and muscle ache }\end{array}$ & $\begin{array}{l}\text { Troponin: } \\
\text { - } \\
\text { NT-proBNP: - }\end{array}$ & $\begin{array}{l}\text { ECG: - } \\
\text { Echo: VT } \\
\text { CMR: Edema+ } \\
\text { Injury- } \\
\text { EMB/PM:NR }\end{array}$ & NR \\
\hline Zeng et al. [47] & Case report & $\begin{array}{l}\text { 63-year-old } \\
\text { man }\end{array}$ & $\begin{array}{l}\text { Shortness of } \\
\text { breath and chest } \\
\text { tightness after } \\
\text { activity }\end{array}$ & $\begin{array}{l}\text { Troponin: } 11.37 \\
\text { g/L } \\
\text { NT-proBNP: } \\
22.600 \mathrm{pg} / \mathrm{mL}\end{array}$ & $\begin{array}{l}\text { ECG: Sinusal tachy } \\
\text { Echo: dyskinesia, } \\
\text { LVD, LVEF } 32 \% \\
\text { CMR: NR } \\
\text { EMB/PM: NR }\end{array}$ & $\begin{array}{l}\text { High-flow oxygen, } \\
\text { CCRTlopinavir-ritonavir, } \\
\text { interferon } \alpha-1 b \text {, } \\
\text { methylprednisolone, } \\
\text { immunoglobulin, } \\
\text { piperacillin-tazobactam }\end{array}$ \\
\hline $\begin{array}{l}\text { Cairns et al. } \\
\text { [48] }\end{array}$ & Case report & $\begin{array}{l}\text { 58-year-old } \\
\text { female }\end{array}$ & $\begin{array}{l}\text { Developed } \\
\text { cardiogenic shock } \\
\text { after fever, } \\
\text { diarrhoea, and } \\
\text { vomiting }\end{array}$ & $\begin{array}{l}\text { Troponin } 3532.9 \\
\text { ng/L } \\
\text { NT-proBNP: NR }\end{array}$ & $\begin{array}{l}\text { ECG: NR } \\
\text { Echo: TTE showed } \\
3-4 \mathrm{~cm} \text { pericardial } \\
\text { effusion with } \\
\text { evidence } \\
\text { of cardiac } \\
\text { tamponade } \\
\text { CMR: NR } \\
\text { EMB/PM: NR }\end{array}$ & $\begin{array}{l}\text { Pericardial drain, } \\
\text { vasopressor support, } \\
\text { intravenous amoxicillin, } \\
\text { oral doxycycline } \\
\text { initially, escalated to } \\
\text { piperacillin/tazobactam, } \\
\text { furosemide, intravenous } \\
\text { immunoglobulin }(2 \mathrm{~g} / \mathrm{kg})\end{array}$ \\
\hline $\begin{array}{c}\text { Beaudry et al. } \\
\text { [49] }\end{array}$ & & $\begin{array}{l}\text { 15-year-old } \\
\text { female }\end{array}$ & $\begin{array}{l}\text { Epigastric pain, } \\
\text { nasal congestion, } \\
\text { and rhinorrhea. } \\
\text { Pyrexia }\left(38.9^{\circ} \mathrm{C}\right) \text {, } \\
\text { tachycardia, and } \\
\text { worsening } \\
\text { respiratory failure }\end{array}$ & $\begin{array}{l}\text { Troponin } \\
2.48 \text { ng/mL } \\
\text { NT-proBNP: } 8328 \\
\text { pg/mL }\end{array}$ & $\begin{array}{l}\text { ECG: sinus } \\
\text { tachycardia } \\
\text { Echo: severely } \\
\text { diminished LV } \\
\text { ejection } \\
\text { CMR: NR } \\
\text { EMB/PM: } \\
\text { cardiomyocytes } \\
\text { with interstitial } \\
\text { lymphoplasmacytic } \\
\text { infiltrate and mixed } \\
\text { inflammation } \\
\text { surrounding } \\
\text { cardiac vessels }\end{array}$ & $\begin{array}{l}\text { Mechanical ventilation } \\
\text { (intensive care unit), } \\
\text { norepinephrine infusion } \\
\text { cefepime, metronidazole, } \\
\text { doxycycline }\end{array}$ \\
\hline
\end{tabular}


Table 1. Cont.

\begin{tabular}{|c|c|c|c|c|c|c|}
\hline Authors & $\begin{array}{l}\text { Study } \\
\text { Design }\end{array}$ & $\begin{array}{c}\text { Sample of } \\
\text { Study }\end{array}$ & $\begin{array}{l}\text { Presenting } \\
\text { Symptoms }\end{array}$ & $\begin{array}{c}\text { Cardiac } \\
\text { Biomarker }\end{array}$ & Results & Treatment \\
\hline $\begin{array}{c}\text { Garot J et al. } \\
{[50]}\end{array}$ & $\begin{array}{l}\text { 69-year-old } \\
\text { man }\end{array}$ & $\begin{array}{c}\text { Fever, asthenia, } \\
\text { and abdominal } \\
\text { pain, } \\
\text { hypotension, } \\
\text { hypoxemia, } \\
\text { anuria, and } \\
\text { multi-organ } \\
\text { failure (MOF) }\end{array}$ & $\begin{array}{l}\text { Troponin } 8066 \\
\text { pg/mL } \\
\text { NT-proBNP: NR }\end{array}$ & & $\begin{array}{l}\text { ECG: no signs of } \\
\text { ischemia } \\
\text { Echo: non-dilated and } \\
\text { severe, diffuse LV } \\
\text { hypokinesia (LV } \\
\text { ejection fraction: 30\%) } \\
\text { CMR: NR } \\
\text { EMB/PM: Abundant } \\
\text { myocardial edema, } \\
\text { interstitial } \\
\text { inflammation } \\
\text { (predominance of } \\
\text { mononucleated } \\
\text { leucocytes) associated } \\
\text { with cardiomyocytes } \\
\text { dystrophies and } \\
\text { strong granular } \\
\text { cytoplasmic staining } \\
\text { of cardiomyocytes for } \\
\text { SARS coronavirus } \\
\text { nucleocapsid protein }\end{array}$ & $\begin{array}{l}\text { Veno-arterial } \\
\text { unit and extracorporeal } \\
\text { membrane oxygenation } \\
\text { (VA-ECMO), } \\
\text { noradrenalin, dobutamine }\end{array}$ \\
\hline
\end{tabular}

Abbreviations: CMR—cardiac magnetic resonance imaging; ECG—electrocardiogram; Echo-echocardiogram; LVH-left ventricular hypertrophy; LVD—left ventricular dilated; NR—not reported; NT-pro-BNP-N-terminal pro B-type natriuretic peptide; EMB-endomyocardial biopsy; PM-post-mortem; ST-ST-segment changes; T-T-wave changes; VT-ventricular tachycardia; CRRT-continuous renal replacement therapy; "+"-abnormal or elevated; "-"-normal.

Table 2. The connection between myocardial injury and COVID-19 as discussed in the relevant studies.

\begin{tabular}{|c|c|c|c|c|c|}
\hline Authors & Study Design & Sample of Study & Results & Treatment & Conclusion \\
\hline Buja et al. [51] & Case series & 23 & $\begin{array}{l}\text { Lymphocytic } \\
\text { myocarditis, } \\
\text { lymphocytic } \\
\text { pericarditis, } \\
\text { multifocal acute } \\
\text { injury of } \\
\text { cardiomyocytes } \\
\text { without } \\
\text { inflammatory } \\
\text { cellular infiltrates }\end{array}$ & $\mathrm{N} / \mathrm{A}$ & $\begin{array}{l}\text { COVID-19 is a } \\
\text { systemic disease } \\
\text { with major } \\
\text { involvement of the } \\
\text { lungs and heart. }\end{array}$ \\
\hline Chen et al. [52] & $\begin{array}{l}\text { Retrospective } \\
\text { study }\end{array}$ & 99 & $\begin{array}{l}\text { Pneumonia, } \\
\text { abnormal CT, } \\
\text { cardiac injury, } \\
\text { secondary } \\
\text { infection }\end{array}$ & $\begin{array}{l}\text { Mechanical respirators } \\
\text { and circulatory } \\
\text { support systems, } \\
\text { including IABP, } \\
\text { Impella, and ECMO }\end{array}$ & $\begin{array}{l}\text { COVID-19 infection } \\
\text { might deteriorate } \\
\text { rapidly, eventually } \\
\text { followed by } \\
\text { fulminant } \\
\text { myocarditis. More } \\
\text { attention should be } \\
\text { paid to patients with } \\
\text { extremely increased } \\
\text { cardiac troponin I } \\
\text { levels and new-onset } \\
\text { arrhythmias. }\end{array}$ \\
\hline Deng et al. [53] & $\begin{array}{l}\text { Retrospective } \\
\text { study }\end{array}$ & 112 & $\begin{array}{l}\text { High troponin } \\
\text { level and } \\
\text { pulmonary } \\
\text { hypertension } \\
\text { indicating } \\
\text { myocardial injury }\end{array}$ & $\mathrm{N} / \mathrm{A}$ & $\begin{array}{l}\text { Myocardial injury is } \\
\text { due to systemic } \\
\text { consequences rather } \\
\text { than direct injury by } \\
\text { Sars-CoV2. }\end{array}$ \\
\hline
\end{tabular}


Table 2. Cont.

\begin{tabular}{|c|c|c|c|c|c|}
\hline Authors & Study Design & Sample of Study & Results & Treatment & Conclusion \\
\hline Escher et al. [54] & $\begin{array}{l}\text { Retrospective } \\
\text { study }\end{array}$ & 104 & $\begin{array}{l}\text { EMB: active } \\
\text { myocarditis } \\
\text { according to the } \\
\text { Dallas criteria, } \\
\text { necrosis }\end{array}$ & $\mathrm{N} / \mathrm{A}$ & $\begin{array}{l}\text { Evidence of } \\
\text { SARS-CoV-2 } \\
\text { genomes detection } \\
\text { in EMB. }\end{array}$ \\
\hline Guo et al. [55] & $\begin{array}{c}\text { Retrospective } \\
\text { single-center case }\end{array}$ & 187 & $\begin{array}{l}\text { Hypertension, } \\
\text { coronary heart } \\
\text { disease, } \\
\text { cardiomyopathy, } \\
\text { elevated TnT level } \\
\text { exhibited } \\
\text { myocardial injury }\end{array}$ & $\begin{array}{l}\text { Antiviral therapy, } \\
\text { antibiotics, respiratory } \\
\text { support }\end{array}$ & $\begin{array}{l}\text { Myocardial injury is } \\
\text { essentially connected } \\
\text { with the lethal } \\
\text { outcome. }\end{array}$ \\
\hline $\begin{array}{l}\text { Halushka et al. } \\
\qquad[56]\end{array}$ & Meta-analysis & $\begin{array}{l}22 \text { studies with } \\
277 \text { patients }\end{array}$ & $\begin{array}{l}\text { Macro or } \\
\text { microvascular } \\
\text { thrombi, } \\
\text { inflammation, or } \\
\text { intraluminal } \\
\text { megakaryocytes }\end{array}$ & $\mathrm{N} / \mathrm{A}$ & $\begin{array}{l}\text { COVID-19-related } \\
\text { cardiac } \\
\text { histopathological } \\
\text { findings are } \\
\text { common, while } \\
\text { myocarditis is rare. }\end{array}$ \\
\hline Han et al. [57] & $\begin{array}{l}\text { Single-center } \\
\text { study }\end{array}$ & 273 & $\begin{array}{l}\text { High blood levels } \\
\text { of CKMB, MYO, } \\
\text { Ultra-TnI, } \\
\text { NTproBNP } \\
\text { indicating acute } \\
\text { cardiac injury }\end{array}$ & $\begin{array}{l}\text { Monitoring of the } \\
\text { myocardiac enzyme } \\
\text { profiles }\end{array}$ & $\begin{array}{l}\text { Higher } \\
\text { concentrations of } \\
\text { CK-MB, MYO, } \\
\text { ultra-TnI, and NT- } \\
\text { proBNPin venous } \\
\text { blood signified the } \\
\text { severity and case } \\
\text { fatality rate (CFR) of } \\
\text { COVID-19. }\end{array}$ \\
\hline Khalid et al. [58] & Case series & 2 & $\begin{array}{l}\text { Abnormal ECG } \\
\text { and Echo, high } \\
\text { blood levels of TnI, } \\
\text { and NTproBNP }\end{array}$ & $\begin{array}{l}\text { Dual antiplatelet } \\
\text { therapy, heparin, } \\
\text { dobutamine, } \\
\text { norepinephrine, } \\
\text { ECMO }\end{array}$ & $\begin{array}{l}\text { Cases of COVID-19 } \\
\text { infection with } \\
\text { myocardial } \\
\text { involvement may } \\
\text { have distinct } \\
\text { mechanistic } \\
\text { pathways and } \\
\text { outcomes. }\end{array}$ \\
\hline $\begin{array}{c}\text { Nunes } \\
\text { Duarte-Neto } \\
\text { et al. [59] }\end{array}$ & Case series & 10 & $\begin{array}{l}\text { Hypertrophy of } \\
\text { cardiomyocytes, } \\
\text { myocardial } \\
\text { fibrosis, interstitial } \\
\text { edema fibrin } \\
\text { thrombi ( } 2 \text { mild } \\
\text { lymphomononu- } \\
\text { clear } \\
\text { myocarditis) }\end{array}$ & $\mathrm{N} / \mathrm{A}$ & $\begin{array}{l}\text { COVID-19 is a } \\
\text { systemic disease } \\
\text { with major effects in } \\
\text { the lungs and the } \\
\text { involvement of } \\
\text { various organs } \\
\text { including the heart. }\end{array}$ \\
\hline $\begin{array}{l}\text { Puntmann et al. } \\
{[60]}\end{array}$ & Cohort study & 100 & $\begin{array}{l}\text { hsTnT values } \\
\text { significantly } \\
\text { elevated, EMB: } \\
\text { active lymphocytic } \\
\text { inflammation, } \\
\text { CMR: myocardial } \\
\text { fibrosis and/or } \\
\text { edema }\end{array}$ & $\begin{array}{l}\text { Antiviral, antibiotic, } \\
\text { steroid, } \\
\text { hydrochloroquine }\end{array}$ & $\begin{array}{l}\text { The combination of } \\
\text { histological findings } \\
\text { as well as the } \\
\text { increase relative to } \\
\text { age-matched, } \\
\text { sex-matched, and } \\
\text { risk-factor-matched } \\
\text { controls makes this a } \\
\text { COVID-19-related } \\
\text { inflammatory } \\
\text { process. }\end{array}$ \\
\hline
\end{tabular}


Table 2. Cont

\begin{tabular}{|c|c|c|c|c|c|}
\hline Authors & Study Design & Sample of Study & Results & Treatment & Conclusion \\
\hline Rajpal et al. [61] & Prospective study & 26 & $\begin{array}{l}\text { CMR: myocardial } \\
\text { edema and } \\
\text { myocardial injury, } \\
\text { pericardial effusion }\end{array}$ & $\mathrm{N} / \mathrm{A}$ & $\begin{array}{l}\text { CMR may provide } \\
\text { an excellent } \\
\text { risk-stratification } \\
\text { assessment for } \\
\text { myocarditis in } \\
\text { athletes who have } \\
\text { recovered from } \\
\text { COVID-19. }\end{array}$ \\
\hline Ruan et al. [62] & $\begin{array}{c}\text { Retrospective } \\
\text { multi-center study }\end{array}$ & 150 & $\begin{array}{l}\text { Predictors of a fatal } \\
\text { outcome included } \\
\text { age, underlying } \\
\text { diseases, } \\
\text { secondary } \\
\text { infection, and } \\
\text { elevated } \\
\text { inflammatory } \\
\text { indicators in the } \\
\text { blood }\end{array}$ & $\mathrm{N} / \mathrm{A}$ & $\begin{array}{l}\text { The infection of } \\
\text { SARS-CoV-2 may } \\
\text { cause fulminant } \\
\text { myocarditis, and } \\
\text { patients with } \\
\text { cardiovascular } \\
\text { diseases have a } \\
\text { significantly } \\
\text { increased risk of } \\
\text { death when they } \\
\text { are infected with } \\
\text { SARS-CoV-2. }\end{array}$ \\
\hline Shi et al. [63] & Cohort study & 416 & $\begin{array}{l}\text { Abnormal ECG, } \\
\text { bilateral } \\
\text { pneumonia, } \\
\text { ground-glass } \\
\text { opacity, mottling }\end{array}$ & $\begin{array}{l}\text { Antiviral therapy, } \\
\text { oxygen, } \\
\text { glucocorticoids, } \\
\text { intravenous } \\
\text { immunoglobulin } \\
\text { therapy, antibiotic } \\
\text { therapy }\end{array}$ & $\begin{array}{l}\text { The data and } \\
\text { findings support } \\
\text { cardiac injury as a } \\
\text { complication in } \\
\text { COVID-19 } \\
\text { patients. }\end{array}$ \\
\hline Yang et al. [64] & Meta-analysis & $\begin{array}{c}6 \text { studies with } 1527 \\
\text { patients }\end{array}$ & $\begin{array}{l}\text { Hypertension, } \\
\text { cardiac } \\
\text { cerebrovascular } \\
\text { disease, and } \\
\text { diabetes }\end{array}$ & $\mathrm{N} / \mathrm{A}$ & $\begin{array}{l}\text { COVID-19 might } \\
\text { aggravate the } \\
\text { damage to the } \\
\text { heart. }\end{array}$ \\
\hline
\end{tabular}

Abbreviations: CMR—cardiac magnetic resonance imaging; ECG-electrocardiogram; Echo-echocardiogram; LVH-left ventricular hypertrophy; LVD—left ventricular dilated; NR—not reported; NT-pro-BNP-N-terminal pro B-type natriuretic peptide; EMB-endomyocardial biopsy; PM-post-mortem; ST-ST-segment changes; T-T-wave changes; VT-ventricular tachycardia; CRRT-continuous renal replacement therapy; "+"-abnormal or elevated; "-"-normal.

In March 2020, two published studies gave the first glimpse of how prevalent heart problems were among patients with COVID-19 disease. The larger of these two studies included 416 hospitalized patients. Among them, a 20\% incidence of heart damage was observed; the mortality reported was $51.2 \%$ in patients with cardiac damage, and $4.5 \%$ in those without it [63].

Similarly, in another study, heart damage occurred in 27.8\% of 187 COVID-19 patients, and the presence of heart damage was associated with fatal outcomes, that is, with a significantly higher mortality (59.6\%) compared to that observed in patients without heart damage $(8.9 \%)$ [55]. In an observational cohort study of German patients who recently recovered from COVID-19 disease and underwent CMRI (with a mean duration of 71 days after COVID-19 diagnosis), 78\% of patients displayed features of heart disease, and $60 \%$ showed signs of ongoing inflammation, regardless of the severity and general course of the disease [60]. This suggests that a potential preexisting SARS-CoV-2 infection should be considered in patients with unexplained acute heart failure or new-onset arrhythmias detected by cardiac electrophysiology procedures. Endomyocardial biopsy (EMB)-based identification of the causes underlying myocardial damage may help explain the diverse evolution of complicated SARS-CoV-2 infection and support the design of future specific and personalized treatment strategies [65-67]. 
The analysis of the available studies highlights a male predominance $(69 \%)$, with the average age of the cases described being 55 years, and with differences between case reports and retrospective studies. COVID-19 patients with myocarditis were $50 \pm 16$ years old, and males and females were equally affected in the case report studies [17-47]. In contrast, retrospective studies described the typical myocarditis patient as male and older than 55 years. One third of all cases were under the age of 40 . Three cases were under the age of 18, with two cases of children under 13 years old. Most patients do not have a previously identified co-morbid condition; hypertension is the most frequently observed risk factor. In general, for these patients, the earliest and most common predominant symptom was dyspnea along with fever, followed by cough and chest pain. Other reported symptoms include fatigue, nausea and vomiting, diarrhea, myalgia, weakness, and headache $[52-55,62]$.

\subsection{ECG Results}

Almost all patients showed ECG features consistent with myocarditis, such as STsegment changes including elevation and depression, T-wave inversions, as well as ventricular tachycardia, nonspecific intraventricular conduction delay, and multiple premature ventricular complexes [52,55,58,60]. Levels of troponin (troponin I or troponin T) were elevated (with remarkably high values in some patients) almost constantly $[21,25,27,29,30,34,37,38,53,57,60]$. N-terminal pro B natriuretic peptide (NT-pro-BNP) was elevated in all cases in which values were measured. Inflammatory markers including white blood cell counts, interleukin-6 levels, and C-reactive protein levels were elevated in all patients (see Tables 1 and 2).

\subsection{Results of Cardiac Ultrasound and Advanced Imaging}

Echocardiography was performed in most cases and revealed a decrease in left ventricular ejection fraction (LVEF). Patients with severely impaired left ventricular function developed cardiogenic shock, and thus, had a worse prognosis. Cardiomegaly or increased wall thickness and pericardial effusion have also been observed, and the presence of cardiac diffuse hypokinesia is registered in $30 \%$ of cases (see Table 1). Cardiac magnetic resonance (CMR) was not performed in all cases, due to the prolonged acquisition time and high contagiousness of COVID-19 infection, but in most cases, myocarditis was diagnosed on the basis of Lake Louise criteria, which included signs of myocardial edema on T2-weighted imaging and myocardial damage on T1-weighted imaging with gadolinium enhancement [19-22,24,25,28,29,32,33,35,37,38,41,42,44-46,60,61]. Bilateral infiltrates and ground glass opacities were observed in all patients undergoing computed tomography (CT) of the chest [52].

\subsection{Histopathological Tests Results}

Few cases of myocarditis diagnosed by histopathological or imaging tests that met the Lake Louise consensus criteria were confirmed in COVID-19 patients [46]. For several others, they remain possible cases, in which the authors reported a diagnosis of myocarditis, with or without support for serological, electrocardiographic, and echocardiographic characteristics. Endomyocardial biopsy (EMB), although considered a gold standard for the diagnostic confirmation of myocarditis, has not been performed in all patients with suspected COVID-19 myocarditis for various reasons, such as the associated coagulopathy and hemodynamic instability. Despite this, in the few cases where it was performed, it showed virus-negative lymphocytic myocarditis, except in seven patients, one from autopsy after sudden death and six from endomyocardial biopsy for clinical reasons. In a patient with hypokinesia and diffuse myocardial edema to the basal and middle segments of the left ventricle on CMR, the EMB showed diffuse inflammatory T-lymphocytic infiltrates. There was massive interstitial edema and limited foci of necrosis, meeting the Dallas criteria. However, the SARS-CoV-2 genome was not identified within the myocardium, suggesting a diagnosis of virus-negative acute lymphocytic myocarditis. A second case was a previously healthy 17-year-old African American male who died on arrival at the hospital after complaining of severe headaches, dizziness, nausea, and vomiting for two days. Pathological 
examination of the heart revealed diffuse inflammatory infiltrates composed of prominent lymphocytes, macrophages, and eosinophils, mainly in the interstitial, and associated with multiple foci of myocyte necrosis. This was identified in both ventricles, and eosinophilic myocarditis was diagnosed. Subsequently, in a study by F. Escher et al., the presence of the SARS-CoV-2 genome was reported in five patients with myocarditis undergoing EMB. From several studies, it is not clear how the viral load or the patient's immunopathology relate to different cardiac pathological findings. Histological and ultrastructural findings in a serious case study reported, from autopsies of mostly elderly fatal cases with significant comorbidities, viral particles (case-to-case variability) found in the heart, large intestine, liver, spleen, kidney, trachea, subcarinal lymph nodes, and cardiac tissue of a patient with lymphocytic myocarditis. The presence of the virus has not always been associated with increased infiltration of mononuclear cells into the myocardium $[23,24,30,41,43,54,56,59]$.

\subsection{Therapies and Advanced Treatment}

Medical management includes therapies targeting COVID-19, therapies for the treatment of myocarditis, and therapies to control the cytokine storm. Corticosteroids were the most used drugs in the treatment attempt, followed by the antiviral drugs lopinavir and ritonavir. The use of hydroxychloroquine, immunoglobulins, piperacillin-tazobactam, and azithromycin was also observed. The use of inotropes and/or vasopressors was reported in $50 \%$ of studies. Additional therapies targeting the cytokine storm, such as tocilizumab and interferon, were also employed. Some patients with COVID-19-induced acute fulminant myocarditis have improved after intravenous glucocorticoid and immunoglobulin therapy. This last finding advocates for the cytokine storm being a result of an inadequate host immune response, which could be the main pathophysiological explanation of COVID-19induced acute fulminant myocarditis. Mechanical support via extracorporeal membrane oxygenation (ECMO) was also used. There is no common consensus for prescribing standard treatment for heart failure in addition to antiviral therapy to hospitalized patients with COVID-19-induced acute myocarditis. In several cases, this last condition was treated with diuretics, angiotensin converting enzyme (ACEi) inhibitors, and beta blockers (see Tables 1 and 2).

\section{Discussion}

\subsection{Biomolecular Hypotheses about COVID-19-Induced Myocardial Injury}

SARS-CoV-2 is a beta coronavirus whose genome consists of single-stranded ribonucleic acid (RNA) with positive polarity that belongs to the Coronaviridae family. The virus can invade the human host cell by binding with high affinity to the angiotensin converting enzyme 2 (ACE 2) receptor. ACE-2 is widely expressed throughout the body, including in the lungs and heart. After penetration, viral RNA enters the cell nucleus for replication and apoptosis $[1,68,69]$. The human immune response to the virus is individual, which explains its variable clinical presentation. The virus mainly infects the lung epithelium with respiratory signs and symptoms, but there have been cases with COVID-19-induced acute myocarditis. The mechanism of heart damage remains poorly understood. Currently, several mechanisms have been proposed to explain the underlying pathophysiology of COVID-19-related acute myocarditis. Among them, the main ones are the following (Figure 1):

(a) Myocardial damage due to the direct viral action on the tissue, or to extrapulmonary migration of infected alveolar macrophages. Thus, a direct myocardial damage through binding to ACE2 receptors because of viral action on the tissue or due to extra pulmonary migration of infected alveolar macrophages. SARS-CoV-2 uses spike protein to bind to ACE2 receptors on the myocardial cell membrane. In theory, following SARS-CoV-2's engagement to the ACE2 receptors on the myocardium and the subsequent down-regulation of the receptors themselves, the accumulation of angiotensin II may enhance myocardial remodeling by diverting all its activity to type 1 angiotensin II receptors (AT1R) of the heart [1,70,71]. Interestingly, a polybasic furin 
site that structurally differentiates SARS-CoV-2 from SARS, when cleaved, expands the types of cells the virus can infect. The virus, by binding to ACE2 receptors throughout the body, facilitates cell entry through its spike protein, together with the cooperation of the serine protease transmembrane protease serine 2, heparan sulfate, and other proteases. The heart is one of the many organs with high ACE2 expression [72-75]. Tropism to other organs besides the lungs was studied from autopsy samples: SARS-CoV-2 genomic RNA was highest in the lungs, but the heart, kidneys, and liver also showed substantial amounts, and copies of the virus were detected in the heart of 16 of the 22 patients who died. In an autopsy series of 39 patients who died from COVID-19, the virus was undetectable in the myocardium in $38 \%$ of patients, while $31 \%$ had a high viral load greater than 1000 copies in the heart $[76,77]$.

(b) Via cell-mediated cytotoxicity in which CD8 T lymphocytes migrate to cardiomyocytes and cause inflammation of the myocardium. Cytokine release syndrome, proposed as the main mechanism underlying COVID-19-induced acute fulminant myocarditis, is thus unleashed [1,9-11]. This triggers the pro-inflammatory cytokine release syndrome, a severe systemic inflammatory response resulting in hypoxia and apoptosis of the cardiomyocytes. In fact, the released cytokines increase the activation of $\mathrm{T}$ lymphocytes, which release further cytokines with consequent positive feedback of immune activation and myocardial damage [9].

(c) The mechanism of hyperactivation of the autoimmune system with possible interferonmediated hyperactivation of the innate and adaptive immune system has also been proposed [78]. Myocarditis can cause the onset of arrhythmias, during the acute phase, due to a direct cytopathic effect, which causes electrical imbalance and ischemia due to microvascular and gap-junctions dysfunction due to reduced myocardial expression of connexins [79]. Thus, the main biomolecular mechanism responsible for heart damage, and in turn heart failure, is an abnormal state of systemic inflammation with low oxygen levels and thrombosis. In addition, the presence of the SARS-CoV-2 genome was detected in the myocardium [54].

Although rare, serious systemic infections such as myocarditis have been reported in connection with COVID-19, myocarditis, an inflammatory condition affecting the myocardium, arises from a broad spectrum of both infectious and non-infectious causes. Many different viruses have been implicated, including the Middle East Respiratory Syndrome (MERS) coronavirus, which closely resembles SARS-CoV-2 [84-87]. Myocarditis is suspected based on elevated troponins in the patient's blood, cardiac arrhythmias or diffuse ST-segment elevation on electrocardiogram (ECG), and impaired contractility of the left ventricular wall (regional or global hypokinesia) on echocardiography. Clinical manifestations of myocarditis include subclinical, subacute, acute, and fulminant forms, and sudden onset myocarditis is known to be associated with significant severity [88-90]. After reviewing the literature and collecting reports related to COVID-19-associated viral myocarditis, it is interesting to note that the diagnosis of viral myocarditis was made on clinical and imaging grounds in several cases of COVID-19. If the predominant injury is caused by widespread inflammation in the context of COVID-19 infection, direct damage to the heart by the virus itself is not excluded.

Some studies have documented the presence of interstitial inflammation of the myocardium and edema on CMRI, while others have reported interstitial inflammatory infiltrates based on EMCB. However, the SARS CoV-2 genome was isolated in very few cases only under EMCB. Therefore, although various non-invasive investigation methods such as echocardiography and nuclear magnetic resonance allow evaluation of the structural and functional alterations of the heart, providing valuable information useful in the diagnosis and prognostic stratification of this disease, the diagnosis of certainty and the complete characterization of the pathological substrate of myocarditis can only be obtained by means of EMCB, an invasive investigation that is critical for planning a specific therapy (see Tables 1 and 2). Nevertheless, the feasibility of performing EMCB in 
all suspected cases is questionable, especially considering the risk of contagious spread, the skill required, and the false negative rate. Furthermore, during this type of pandemic, it is not feasible to perform an EMCB only to confirm or rule out acute viral myocarditis in patients with signs and symptoms of acute heart failure, even based on suggestive features by CMRI, and without other plausible causes. This limitation has been partially overcome, where possible, with autopsies in patients with suspected Covid-19 myocarditis to detect the presence of viral genomes. To date, although clinical data on myocarditis caused by SARS-CoV-2 are limited, patient characteristics, clinical course, degree of severity, imaging and electrocardiographic hallmarks, and management and outcomes of myocarditis are particularly relevant, and should be considered by physicians when facing patients with potential COVID-19 myocarditis. However, the potential pathogenetic significance of this finding is still unclear $[54,70,91-95]$.

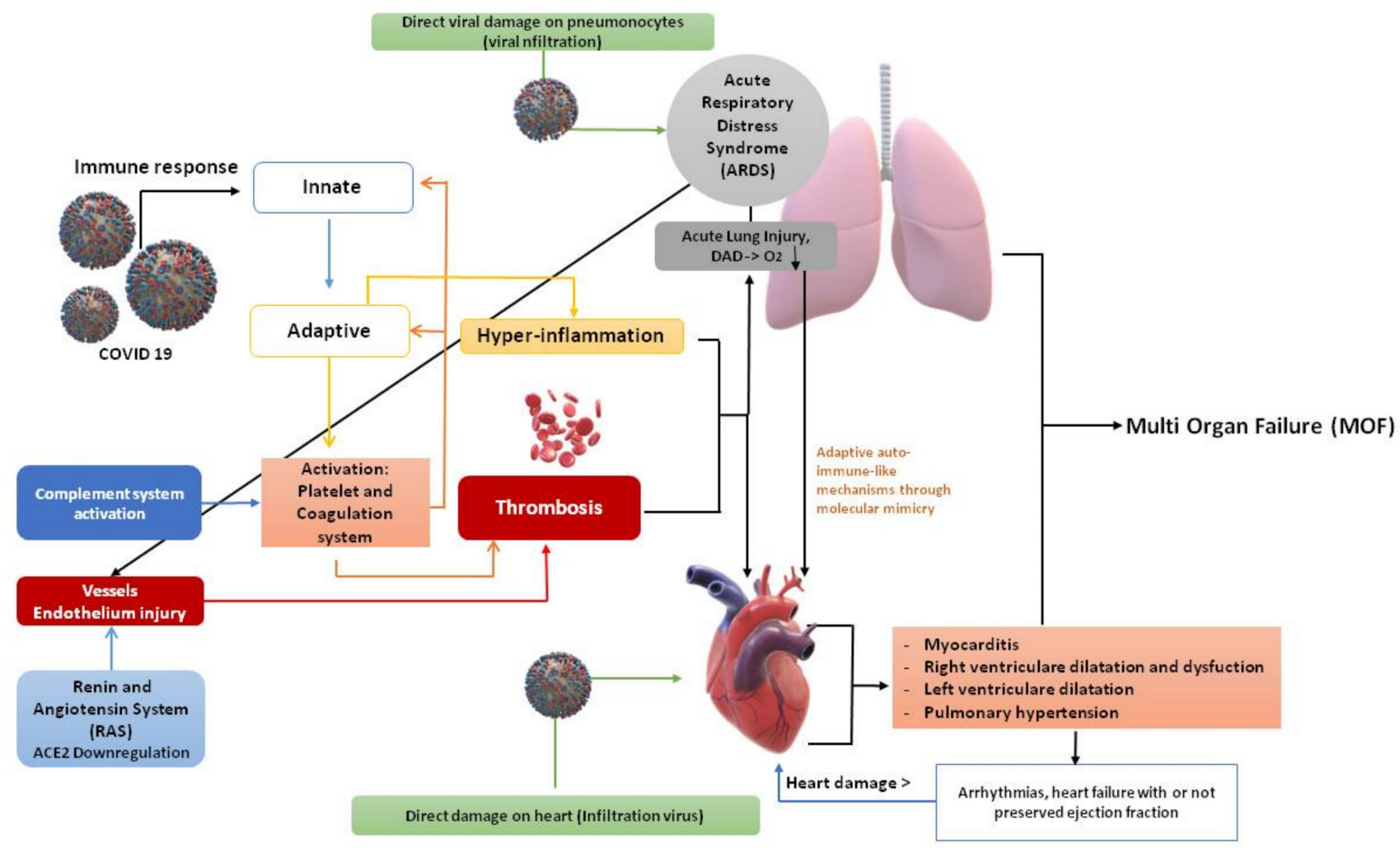

Figure 1. The myocardial damage hypothesis. Although the predominant injury can be ascribed to the wide-spreading inflammation in the context of COVID-19 infection, a direct heart damage by the virus itself is not excluded. According to this hypothesis, SARS-CoV-2 can directly and indirectly affect both pneumocytes and myocardiocytes, concomitantly leading to a severe injury of lung and heart tissues. The initial activation of the innate immune response, and the subsequent recruitment of the adaptive immune system (CD8 and B antiviral cells, antibodies) with the contribution of the complement system (such as $\mathrm{C} 3 \mathrm{a}, \mathrm{C} 5 \mathrm{a}$, membrane complex) may explain the hyper-inflammation (by cytokines/chemokines storm) and its deleterious consequences for lung and heart tissues. In addition, downregulation of ACE2 receptors increases ACE/AT1, which leads in turn to vasoconstriction and endothelial damage in both lung and heart. Activation of the coagulation system and dysregulation of ACE2 will also amplify the risk of clot formation, with enhanced thrombosis due to a hypercoagulable state. This condition increases the possibility of diffuse alveolar damage (DAD), aggravates the severe hypoxemia, and triggers an acute respiratory distress syndrome (ARDS) on one side, but may also aggravate an underlying heart dysfunction and induce myocardial ischemia and heart damage. Severe hypoxemia may progressively worsen the harmed myocardium and result in acute myocarditis, dilatation of the left and/or right ventricle (in this case leading to pulmonary hypertension), or impaired electrophysiological and structural heart remodeling. The co-existence of myocardial inflammation and interstitial edema with cardiac fibrosis, together with an abnormal regulation of the intracellular $\mathrm{Ca}^{++}$and $\mathrm{K}^{+}$concentrations can facilitate arrhythmias (especially tachyarrhythmias) and subsequently heart failure, further damaging the heart. In the worst scenario, the overall systemic damage may lead to a multi-organ failure (MOF) $[1,9,78,80-83]$ Credits: Original figure by I.A. Charitos). 


\subsection{Cardiovascular Signs and Symptoms during the Infection}

A variety of cardiac disturbances have been observed in the COVID-19 pandemic, including acute myocardial infarction, acute heart failure, cardiogenic shock, myocarditis, and malignant arrhythmias. It must be emphasized that the viral infection can not only deregulate a pre-existing heart disease, but also exacerbate mild asymptomatic pre-existing heart failure with long-term complications. The increased risk of complications in patients with heart failure, various arrhythmias, post-infarction, and similar vulnerable conditions leads to a higher risk for suspected myocardial damage (Figure 2) [81-83].

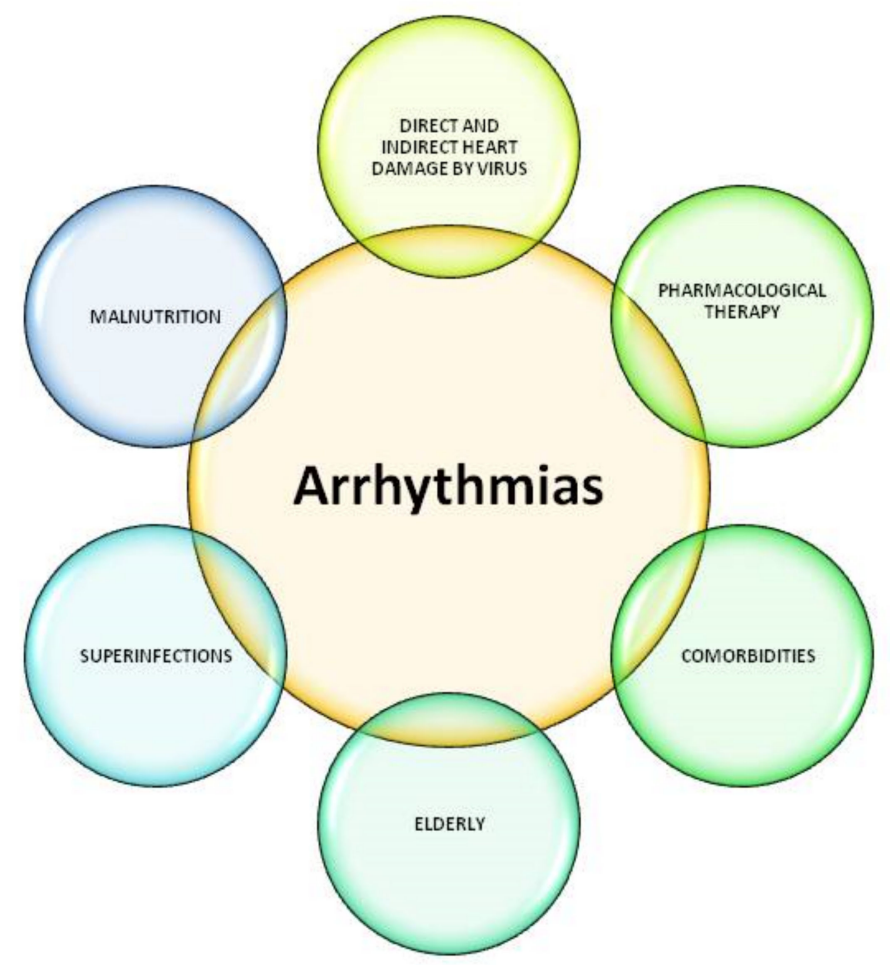

Figure 2. The main modifiable and non-modifiable risk factors that may predispose patients to arrhythmias. Several arrhythmias may arise in patients with myocarditis during SARS CoV-2 infection, especially in the critical care setting. Among them, both bradyarrhythmias (such as atrioventricular nodal block, sinus node dysfunction, and sinus bradycardia) and tachyarrhythmias (such as ventricular tachycardia, atrial fibrillation, atrial flutter, atrial tachycardia, sinus tachycardia) may occur. Inflammatory cytokines acting on both cardiomyocytes (especially interleukin 6) and autonomic nervous system (ANS) can promote QTc prolongation. The administration of antivirals (such as protease inhibitors lopinavir/ritonavir), antibiotics (such as macrolides, fluoroquinolones, and others), and the antimalarials (such as hydroxychloroquine) can trigger similar consequences. Finally, cardiometabolic risk factors (such as obesity, diabetes), recent or existing cardiac diseases (previous myocardial injury, atrial fibrillation, hypertensive heart disease, and others), and chronic pneumopathies (chronic obstructive pulmonary disease (COPD) and others) are examples of the comorbidities that increase the risk of heart damage and therefore favor the onset of malignant arrhythmias.

Patients with underlying cardiovascular disease (CVD) have an increased risk of developing heart damage $[6,74,75]$. In a retrospective single-center study that included 187 patients, the mortality rate was higher in patients with underlying CVD than in patients without CVD (54.5\% vs. 13.2\%) [55]. In order of prevalence, the clinical manifestations of severe acute respiratory syndrome due to COVID-19 are fever, cough, and fatigue [6]. Typically, myocarditis arises with fever, dyspnea, and chest pain, all symptoms which hinder the recognition and clinical diagnosis of myocarditis during the COVID-19 pandemic $[7,96]$. Indeed, our systematic revision of selected studies confirms that in patients with COVID-19, myocarditis commonly starts with dyspnea, followed by cough, fever, 
and chest pain. Other reported symptoms include fatigue, nausea and vomiting, diarrhea, myalgia, weakness, and headache.

\subsection{Main Laboratory Markers of Cardiovascular Severe Prognosis}

Serum biomarkers are conventionally used to confirm suspected acute myocarditis. While troponin was elevated in some patients with acute myocarditis, elevated serum cardiac troponins were almost always seen in fulminant myocarditis. Elevated troponin levels have been described in many COVID-19 patients, with differences observed between deceased and surviving patients. The absence of an increase in serum cardiac troponins, however, does not rule out myocarditis $[6,7,78,83]$. The retrospective study of a single Wuhan center that included 187 patients showed that $37.5 \%$ of deceased patients had heart damage with elevated troponin, and mortality was $69.4 \%$ in those with a history of CVD [55]. In most studies, cardiac damage was associated with elevated troponin and pro-BNP amounts. Levels of these markers were higher in critically ill patients admitted to intensive care. Levels of creatine kinase myocardial band (CKMB) and BNP were often elevated in myocarditis and can provide information on prognosis. CRP was commonly elevated, although normal levels did not exclude myocarditis. However, CRP has also been shown to be above the normal range in most COVID-19 patients, and increases in troponins, $\mathrm{CK}-\mathrm{MB}$, and $\mathrm{BNP}[7,78,83]$. It is important to underline an aspect that represents a limitation in the differential diagnosis of COVID-19 myocarditis. Troponin has been shown as an independent predictor of mortality in hospitalized patients with COVID-19. In COVID-19 patients who have elevated troponin levels, it may be difficult to reach a definitive diagnosis of acute coronary syndrome or acute myocarditis because there are similarities between them regarding elevated troponin levels and changes in ECG. It is reasonable not to perform MRI in most patients due to the contiguity of COVID-19. On the other hand, the underuse of the modalities in the differential diagnosis reveals a gap in the identification of the definitive etiology of myocardial damage (see Tables 1 and 2). Therefore, we may underestimate the prevalence and importance of COVID-19-related myocarditis. Additionally, in severe patients, both acute coronary syndrome (ACS) and acute myocarditis can occur together due to the procoagulant and inflammatory nature of the COVID-19 infection [1,6,7,78,97].

Finally, some studies report that the laboratory results showed differences in white blood cell counts and specifically in lymphocytes count. Indeed, in a retrospective multicenter study for clinical predictors of mortality in patients who had died $(\mathrm{N}=68)$, white blood cells $(\times 109 / 1)$ were 10.62 with lymphocyte counts $(\times 109 / 1) 0.60(0.32)$; instead, in the discharged patients $(\mathrm{N}=82)$ white blood cells were 6.76 (3.49) and lymphocytes 1.42 (2.14), with a $p$-value $<0.001$ for both two groups. In another retrospective single-center case study with 187 patients, it was found that Troponin T levels and NT-proBNP levels were significantly increased in deceased patients. These patients had higher white blood cell and neutrophil counts and lower lymphocyte counts than those with normal Troponin T levels $[54,55,62]$. Therefore, there may be a correlation between lymphopenia and myocarditis severity, but this needs further investigation to confirm it.

\subsection{Instrumental Diagnostics}

ECGs of most patients with myocarditis have demonstrated nonspecific features including sinus tachycardia, ST-segment, and T-wave abnormalities, and occasional atrioventricular or branch blocks. Although ST-segment elevation in segmentally contiguous leads or in a non-vascular distribution are common electrocardiographic signs of myocarditis, they can often be mistaken for coronary occlusion $[7,74,83,96,98]$. Based on studies reported here, ECG changes were non-specific and highly variable. This is consistent with the non-specific ECG findings in myocarditis reported in the literature. Serial ECGs can be a tool to provide a relatively quick, affordable, and non-invasive means of delineating and intervening in the early stages of the disease process (see Tables 1 and 2). 
Echocardiography is important in the assessment of structural and functional changes secondary to myocarditis. Although there are no specific echocardiographic features of myocarditis, it might nevertheless allow the physician to rule out other causes of heart failure, including pericardial effusion, and intracavitary thrombi [98-101].

Cardiac MRI is the non-invasive gold standard test for myocarditis. It was performed in a few of the reported cases, all of which showed an increase in signal intensity after gadolinium. Some showed evidence of myocarditis as fulfilled by the Lake Louis criteria for the diagnosis of myocarditis, such as edema and myocardial damage $[60,101]$.

\subsection{Histological Findings of Myocarditis}

In histological diagnosis of myocarditis, echocardiographic patterns of dilated, hypertrophic, restrictive, and ischemic cardiomyopathies have been described, especially left ventricular dilation, and increase in volume. The reduction in LVEF is an independent predictor of a fulminant disease course [7,102]. Some reviews report that most patients with COVID-19 myocarditis had reduced LVEF, cardiomegaly, and pleural effusion. Dilated ventricles are typical of acute myocarditis, although the fulminant form can lead to an increase in the thickness of the left ventricular wall because of active inflammation [102]. Endomyocardial biopsy (EMB), although considered a gold standard for the diagnostic confirmation of myocarditis, has not been performed in all patients with suspected COVID19 myocarditis for various reasons, but remains the key test to detect the presence of the SARS-CoV-2 genome (see Tables 1 and 2).

\subsection{Management and Treatment Strategies}

Among COVID-19 patients, there is limited evidence of the effectiveness of current management and treatment strategies [6]. Several treatments have been used in COVID-19 myocarditis based on our understanding of the pathogenesis and previous experience in the treatment of viral and fulminant myocarditis. Since cytokine release syndrome is a probable mechanism of injury in COVID-19 myocarditis, glucocorticoids have been used despite the lack of proven clinical efficacy $[103,104]$. Data on the use of glucocorticoids in COVID-19 infection appear controversial. In a Wuhan study involving 84 patients with ARDS secondary to COVID-19, administration of corticosteroids reduced the risk of mortality. Additionally, in a recent press release from RECOVERY (Randomized Evaluation of COVid-19 thERapY), a large clinical trial in COVID-19 patients, dexamethasone was shown to reduce mortality in one-third of ventilated patients. In a recent review of COVID-19associated myocarditis, a study was reported in which five out of seven patients who had been treated with corticosteroids recovered; one died, and the other case reported no results. We cannot confirm if it's due to a true treatment effect or just chance, but based on recent data, the steroid may be associated with favorable outcomes in critically ill COVID-19 patients [105-107]. Some patients with COVID-19-induced acute fulminant myocarditis have shown improvement using intravenous (IV) administration of glucocorticoid and immunoglobulin therapy. Combined steroid treatment with immunomodulatory therapy and antibiotics was used in a minority of studies. IV immunoglobulin (IG) administration has been used with inconsistent outcomes. There is strong evidence for the efficacy of IV-IG in the treatment of acute myocarditis. A meta-analysis published in 2019 comparing IV-IG with corticosteroids for acute myocarditis showed that IV-IG therapy improved mortality and recovery of left ventricular function. There are only a few blinded randomized prospective studies that have specifically targeted viral infections or inflammatory cascades associated with myocarditis. Currently, the evidence does not support the routine use of IV corticosteroids or IV-IGs alone [108-110].

Tocilizumab is an IL-6 receptor antagonist used most in the treatment of rheumatoid arthritis. It is also approved for use in cytokine release syndrome based on multiple studies that have shown its effectiveness. In several cases on different studies examined it gave erratic results. 
Other possible treatments are plasma exchange therapy, immunosuppression with IV-IG and cytokine inhibitors, and antiviral agents such as remdesivir. In the early results of the studies, remdesivir was found to be superior to placebo in shortening healing time in adults hospitalized with COVID-19. Until prospective studies and trials establish guidelines for the management of COVID-19 myocarditis, treatment must be tailored to the presentation of individual cases [110-113].

The guidelines of the European Society of Cardiology Working Group on Myocardial and Pericardial Diseases recommend optimal management of heart failure and arrhythmias caused by myocarditis, including the use of diuretics, ACE inhibitors, beta-adrenergic blockade, antiarrhythmics, and temporary stimulation [114].

The use of extracorporeal membrane oxygenation has been shown to provide favorable outcomes for patients with myocarditis and sepsis, as well as fulminant myocarditis [115-117].

Immunosuppression with the use of steroids, azathioprine, and cyclosporine is often attempted, but guidelines recommend starting only after active polymerase chain reaction infections have been established $[6,7,118]$.

At present, given the lack of results from randomized control studies, the effectiveness of the various treatment strategies currently attempted in cases of COVID-19 myocarditis remains uncertain.

\subsection{Knowledge Gaps and Future Directions}

Due to the heterogeneous and not always consistent results from clinical studies, the current guideline recommendations on the most appropriate clinical management in patients diagnosed with myocarditis are extremely general. The intriguing question on why some individuals may possess a higher predisposition to develop a heart involvement after SARS-CoV-2 infection is still unanswered. A few months after the pandemic began, a cardiac involvement would be predicted mainly in patients with severe COVID-19 infection. However, present data suggest that this condition is more common than expected, although the exact incidence is unknown. It is therefore crucial to determine the most important factors involved in the pathogenesis of myocarditis and to clarify whether it may develop as an individual inflammatory response, an autoimmune phenomenon, or for some other reasons still unexplored. If preventing SARS-CoV-2 infections is crucial, the goal of averting cardiovascular involvement is not secondary. To this aim, it is important to have a low threshold of suspicion in the presence of clinical syndromes of recent onset, and immediately proceed to the precise and exhaustive characterization of the myocardial substrate by taking advantage of the tools available today. Recent study data show heart consequences such as myocarditis after recovery in patients who had no cardiac symptoms during their illness. In fact, in a study using cardiac magnetic resonance, it was observed that out of one hundred patients recovered from COVID-19 (treated at home or in the hospital), residual cardiac inflammation remained in 53\% of patients. Another study considered 26 patients without comorbidity (professional athletes), of which 12 had mild symptoms, while 14 were asymptomatic. It was noted that four patients detected myocarditis without clinical symptoms, and two with mild symptoms, such as shortness of breath. Finally, none of the patients had an electrocardiogram, a blood test (troponin levels), or anything else that can show heart pain or other symptoms. It remains to be clarified when the inflammation of the myocardium develops after the exposure to coronavirus and when it remains active. This condition could compromise returning to normal physical activity after COVID-19 for a few months. According to the CDC (Centers for Disease Control and Prevention, USA), although most people with COVID-19 get better within weeks of the illness, some people experience post-COVID conditions (see Table 3) [119-123].

Thus, in the management of a patient with myocarditis, the follow-up of the clinicalinstrumental picture and the reassessment of the ongoing evolution in the short and medium term are fundamental both for an accurate prognostic stratification in the long term and for the early identification of cases with a more negative natural history. Indeed, 
research is currently focusing on the long-term consequences after the viral infection is resolved.

Table 3. Health Issues (post-COVID 19 conditions) for infected individuals according to CDC (modified from source: https: / /www.cdc.gov / coronavirus/2019-ncov/long-term-effects.html (accessed on 20 May 2021).

\begin{tabular}{|c|c|c|}
\hline \multicolumn{3}{|c|}{ Types of Post-COVID Conditions } \\
\hline Long COVID-19 & $\begin{array}{c}\text { COVID-19 } \\
\text { Multiorgan Effects }\end{array}$ & $\begin{array}{c}\text { COVID-19 } \\
\text { Treatment or Hospitalization Effects }\end{array}$ \\
\hline $\begin{array}{l}\text { A range of symptoms that can last weeks } \\
\text { or months after first being infected or can } \\
\text { appear weeks after infection. Can happen } \\
\text { to anyone who has had COVID-19, even } \\
\text { if the illness was mild, or they had no } \\
\text { symptoms. Tiredness or fatigue, difficulty } \\
\text { thinking or concentrating (sometimes } \\
\text { referred to as "brain fog"), headache, loss } \\
\text { of smell or taste, orthostatic dizziness, } \\
\text { fast-beating or pounding heart (also } \\
\text { known as heart palpitations), chest pain, } \\
\text { difficulty breathing or shortness of breath, } \\
\text { cough, joint or muscle pain, depression or } \\
\text { anxiety, fever, symptoms that get worse } \\
\text { after physical or mental activities. }\end{array}$ & $\begin{array}{l}\text { Multiorgan effects can affect most, if not } \\
\text { all, body systems, including heart, lung, } \\
\text { kidney, skin, and brain functions. Can } \\
\text { also include conditions that occur after } \\
\text { COVID-19, like multisystem } \\
\text { inflammatory syndrome (MIS) and } \\
\text { autoimmune condition. }\end{array}$ & $\begin{array}{l}\text { Longer-term effects of COVID- } 19 \\
\text { treatment or hospitalization (some of } \\
\text { these effects are similar to those related to } \\
\text { hospitalization for other respiratory } \\
\text { infections or other conditions). Can } \\
\text { include severe weakness and } \\
\text { post-traumatic stress disorder (PTSD } \\
\text { involves long-term reactions to a stressful } \\
\text { event), and post-intensive care syndrome } \\
\text { (PICS). }\end{array}$ \\
\hline
\end{tabular}

\subsection{Limitations}

An authentic estimate of myocarditis in patients with COVID-19 infection is limited by several factors. First, given the profound impact of the COVID-19 pandemic on routine clinical practice and diagnostic approaches, several patients did not undergo further diagnostic investigations by means of cardiac magnetic resonance and/or endomyocardial biopsy. Second, it is important to emphasize that echographic findings are often unspecific, and abnormalities in wall motility as well as impaired ventricular function may be observed in cases of stress cardiomyopathy not necessarily dependent on viral infection. In addition, publication bias by the authors reporting some cases should also be taken into consideration. Finally, the patient number turns out to be a limitation. This is because the results in this review are based on conclusions from selected literature, which are thus lacking statistical significance because they are a simple cumulation of observations made in different contexts. However, and despite these limitations, data illustrated in this review should provide useful information for detecting trends and help making new assumptions on the potential onset of myocarditis in subjects with SARS-CoV-2 infection.

\section{Conclusions}

Our knowledge on management of COVID-19 infection is growing exponentially. Myocardial damage and myocarditis have been shown to be associated with increased morbidity and mortality, but there are still many unanswered questions and several areas to explore. Currently, most of the ongoing research focuses on respiratory complications, and little is known about the aspects characterizing COVID-19 myocarditis. Cases referred to previously considered healthy patients who have experienced heart failure linked to myocarditis, associated with SARS-CoV2 positivity, are growing. Nevertheless, data are, at present, still insufficient to explain how the new coronavirus should damage the heart muscle, how frequent is the heart inflammation associated with COVID-19 infection, and whether the available therapeutic strategy might be applied to all patients. In addition, several difficulties might be encountered in the differential diagnosis of acute myocarditis in the context of COVID-19 with respect to the SARS-CoV2-dependent myocarditis correlation. These uncertainties may explain the current lack of consensus on diagnostic and treatment algorithms in patients with a suspected COVID-19-induced acute myocarditis. 
By recapitulating the characteristics of the main studies that have analyzed COVID-19 patients with myocarditis, this review highlights the need for further research to improve our understanding of this pathological process and help define the most appropriate approach in these patients.

Author Contributions: All authors contributed to the study conception and design. Literature search, data collection, and analysis were performed by A.M.S., I.A.C., and L.S. Critical revision of the manuscript for important intellectual content has been done by D.M.C., D.D., and M.M. R.L., S.T. and A.E.W. provided resources and statistical analysis. D.M.C. and L.S. supervised the project. The first draft of the manuscript was written by A.M.S., and all authors revised and commented on previous versions of the manuscript. All authors have read and agreed to the published version of the manuscript.

Funding: This research has been funded by Accademia Italiana Medici Specializzandi (AIMS). The funder had no influence in the content and conclusion of the study.

Institutional Review Board Statement: Not applicable.

Informed Consent Statement: Not applicable.

Data Availability Statement: Not applicable.

Acknowledgments: The authors thanks Giuseppe Divittorio and Michelangelo Ferri, Laboratory Technicians at Microbiology and Virology Unit of Policlinico University Hospital of Bari, for their support.

Conflicts of Interest: The authors report no relationships that could be construed as a conflict of interest.

\section{References}

1. Santacroce, L.; Charitos, I.A.; Carretta, D.M.; De Nitto, E.; Lovero, R. The human coronaviruses (HCoVs) and the molecular mechanisms of SARS-CoV-2 infection. J. Mol. Med. 2021, 93-106. [CrossRef]

2. Wang, C.; Horby, P.W.; Hayden, F.G.; Gao, G.F. A novel coronavirus outbreak of global health concern. Lancet 2020, 395, 470-473. [CrossRef]

3. Santacroce, L.; Charitos, I.A.; Del Prete, R. COVID-19 in Italy: An Overview from the First Case to Date. Electron. J. Gen. Med. 2020, 17, em235. [CrossRef]

4. World Health Organization. Coronavirus. Available online: https://www.who.int/health-topics/coronavirus (accessed on 1 December 2020).

5. $\quad$ Li, S.; Li, S.; Disoma, C.; Zheng, R.; Zhou, M.; Razzaq, A.; Liu, P.; Zhou, Y.; Dong, Z.; Du, A.; et al. SARS-CoV-2: Mechanism of infection and emerging technologies for future prospects. Rev. Med. Virol. 2021, 31, e2168. [CrossRef]

6. Charitos, I.A.; Ballini, A.; Bottalico, L.; Cantore, S.; Passarelli, P.C.; Inchingolo, F.; D'Addona, A.; Santacroce, L. Special features of SARS-CoV-2 in daily practice. World J. Clin. Cases 2020, 8, 3920-3933. [CrossRef] [PubMed]

7. Wujtewicz, M.; Dylczyk-Sommer, A.; Aszkiełowicz, A.; Zdanowski, S.; Piwowarczyk, S.; Owczuk, R. COVID-19-What should anaethesiologists and intensivists know about it? Anaesthesiol. Intensive Ter. 2020, 52, 34-41. [CrossRef]

8. Santacroce, L.; Bottalico, L.; Charitos, I.A. The Impact of COVID-19 on Italy: A Lesson for the Future. Int. J. Occup. Environ. Med. 2020, 11, 151-152. [CrossRef]

9. Gralinski, L.E.; Baric, R.S. Molecular pathology of emerging coronavirus infections. J. Pathol. 2015, 235, 185-195. [CrossRef]

10. Cazzolla, A.P.; Lovero, R.; Muzio, L.L.; Testa, N.F.; Schirinzi, A.; Palmieri, G.; Pozzessere, P.; Procacci, V.; Di Comite, M.; Ciavarella, D.; et al. Taste and Smell Disorders in COVID-19 Patients: Role of Interleukin-6. Acs Chem. Neurosci. 2020, 11, $2774-2781$. [CrossRef]

11. Fox, S.E.; Akmatbekov, A.; Harbert, J.L.; Li, G.; Brown, J.Q.; Heide, R.S.V. Pulmonary and Cardiac Pathology in Covid-19: The First Autopsy Series from New Orleans. medRxiv 2020. [CrossRef]

12. Mohanty, S.K.; Satapathy, A.; Naidu, M.M.; Mukhopadhyay, S.; Sharma, S.; Barton, L.M.; Stroberg, E.; Duval, E.J.; Pradhan, D.; Tzankov, A.; et al. Severe acute respiratory syndrome coronavirus-2 (SARS-CoV-2) and coronavirus disease 19 (COVID-19)Anatomic pathology perspective on current knowledge. Diagn. Pathol. 2020, 15, 103. [CrossRef] [PubMed]

13. Atri, D.; Siddiqi, H.K.; Lang, J.; Nauffal, V.; Morrow, D.A.; Bohula, E.A. COVID-19 for the cardiologist: A current review of the virology, clinical epidemiology, cardiac and other clinical manifestations and potential therapeutic strategies. J. Am. Coll. Cardiol. Basic Transl. Sci. 2020, 5, 518-536. [CrossRef]

14. Schirinzi, A.; Cazzolla, A.P.; Lovero, R.; Muzio, L.L.; Testa, N.F.; Ciavarella, D.; Palmieri, G.; Pozzessere, P.; Procacci, V.; Di Serio, F.; et al. New Insights in Laboratory Testing for COVID-19 Patients: Looking for the Role and Predictive Value of Human epididymis secretory protein 4 (HE4) and the Innate Immunity of the Oral Cavity and Respiratory Tract. Microorganisms 2020, 8, 1718. [CrossRef] 
15. Alhogbani, T. Acute myocarditis associated with novel Middle East respiratory syndrome coronavirus. Ann. Saudi Med. 2016, 36, 78-80. [CrossRef] [PubMed]

16. Babapoor-Farrokhran, S.; Gill, D.; Walker, J.; Rasekhi, R.T.; Bozorgnia, B.; Amanullah, A. Myocardial injury and COVID-19: Possible mechanisms. Life Sci. 2020, 253, 117723. [CrossRef]

17. Asif, T.; Ali, Z. Transient ST Segment Elevation in Two Patients with COVID-19 and a Normal Transthoracic Echocardio-gram. Eur. J. Case Rep. Intern. Med. 2020, 7. [CrossRef]

18. Beri, A.; Kotak, K. Cardiac injury, arrhythmia, and sudden death in a COVID-19 patient. HeartRhythm Case Rep. 2020, 6, 367-369. [CrossRef] [PubMed]

19. Bernal-Torres, W.; Herrera-Escandón, Á.; Hurtado-Rivera, M.; Plata-Mosquera, C.A. COVID-19 fulminant myocarditis: A case report. Eur. Heart J. Case Rep. 2020, 4, 1-6. [CrossRef] [PubMed]

20. Beşler, M.S.; Arslan, H. Acute myocarditis associated with COVID-19 infection. Am. J. Emerg. Med. 2020, 38, 2489.e1-2489.e2. [CrossRef]

21. Cizgici, A.Y.; Agus, H.Z.; Yildiz, M. COVID-19 myopericarditis: It should be kept in mind in today's conditions. Am. J. Emerg. Med. 2020, 38, 1547.e5-1547.e6. [CrossRef]

22. Coyle, J.; Igbinomwanhia, E.; Sanchez-Nadales, A.; Danciu, S.; Chu, C.; Shah, N. A Recovered Case of COVID-19 Myocarditis and ARDS Treated With Corticosteroids, Tocilizumab, and Experimental AT-001. JACC Case Rep. 2020, 2, 1331-1336. [CrossRef]

23. Craver, R.; Huber, S.; Sandomirsky, M.; McKenna, D.; Schieffelin, J.; Finger, L. Fatal Eosinophilic Myocarditis in a Healthy 17-Year-Old Male with Severe Acute Respiratory Syndrome Coronavirus 2 (SARS-CoV-2c). Fetal Pediatr. Pathol. 2020, 39, 263-268. [CrossRef]

24. Dolhnikoff, M.; Ferranti, J.F.; Monteiro, R.A.D.A.; Duarte-Neto, A.N.; Gomes-Gouvêa, M.S.; Degaspare, N.V.; Delgado, A.F.; Fiorita, C.M.; Leal, G.N.; Rodrigues, R.M.; et al. SARS-CoV-2 in cardiac tissue of a child with COVID-19-related multisystem inflammatory syndrome. Lancet Child Adolesc. Health 2020, 4, 790-794, Erratum in 2020, 4, e39, doi:10.1016/s2352-4642(20)30257-1. [CrossRef]

25. Doyen, D.; Moceri, P.; Ducreux, D.; Dellamonica, J. Myocarditis in a patient with COVID-19: A cause of raised troponin and ECG changes. Lancet 2020, 395, 1516. [CrossRef]

26. Fried, J.A.; Ramasubbu, K.; Bhatt, R.; Topkara, V.K.; Clerkin, K.J.; Horn, E.; Rabbani, L.; Brodie, D.; Jain, S.S.; Kirtane, A.J.; et al. The Variety of Cardiovascular Presentations of COVID-19. Circulation 2020, 141, 1930-1936. [CrossRef] [PubMed]

27. Hu, H.; Ma, F.; Wei, X.; Fang, Y. Coronavirus fulminant myocarditis treated with glucocorticoid and human immunoglobulin. Eur. Heart J. 2021, 42, 206. [CrossRef] [PubMed]

28. Inciardi, R.M.; Lupi, L.; Zaccone, G.; Italia, L.; Raffo, M.; Tomasoni, D.; Cani, D.S.; Cerini, M.; Farina, D.; Gavazzi, E.; et al. Cardiac Involvement in a Patient With Coronavirus Disease 2019 (COVID-19). JAMA Cardiol. 2020, 5, 819-824. [CrossRef]

29. Irabien-Ortiz, Á.; Carreras-Mora, J.; Sionis, A.; Pàmies, J.; Montiel, J.; Tauron, M. Fulminant myocarditis due to COVID-19. Rev. Española Cardiol. (Engl. Ed.) 2020, 73, 503-504. [CrossRef]

30. Jacobs, J.P.; Stammers, A.H.; Louis, J.S.; Hayanga, J.W.A.; Firstenberg, M.S.; Mongero, L.B.; Tesdahl, E.A.; Rajagopal, K.; Cheema, F.H.; Coley, T.; et al. Extracorporeal Membrane Oxygenation in the Treatment of Severe Pulmonary and Cardiac Compromise in Coronavirus Disease 2019: Experience with 32 Patients. ASAIO J. 2020, 66, 722-730. [CrossRef] [PubMed]

31. Juusela, A.; Nazir, M.; Gimovsky, M. Two cases of coronavirus 2019-Related cardiomyopathy in pregnancy. Am. J. Obstet. Gynecol. MFM 2020, 2, 100113. [CrossRef] [PubMed]

32. Kim, I.-C.; Kim, J.Y.; Kim, H.A.; Han, S. COVID-19-related myocarditis in a 21-year-old female patient. Eur. Heart J. 2020, 41, 1859. [CrossRef]

33. Luetkens, J.A.; Isaak, A.; Zimmer, S.; Nattermann, J.; Sprinkart, A.M.; Boesecke, C.; Rieke, G.J.; Zachoval, C.; Heine, A.; Velten, M.; et al. Diffuse Myocardial Inflammation in COVID-19 Associated Myocarditis Detected by Multiparametric Cardiac Magnetic Resonance Imaging. Circ. Cardiovasc. Imaging 2020, 13, e010897. [CrossRef]

34. Naneishvili, T.; Khalil, A.; O'Leary, R.; Prasad, N. Fulminant myocarditis as an early presentation of SARS-CoV-2. BMJ Case Rep. 2020, 13, e237553. [CrossRef]

35. Oberweis, M.-L.; Codreanu, A.; Boehm, W.; Olivier, D.; Pierron, C.; Tsobo, C.; Kohnen, M.; Abdelrahman, T.T.; Nguyen, N.T.; Wagner, K.; et al. Pediatric Life-Threatening Coronavirus Disease 2019 With Myocarditis. Pediatr. Infect. Dis. J. 2020, 39, e147-e149. [CrossRef] [PubMed]

36. Oleszak, F.; Maryniak, A.; Botti, E.; Abrahim, C.; Salifu, M.O.; Youssef, M.; Henglein, V.L.; McFarlane, S.I. Myocarditis Associated With COVID-19. Am. J. Med. Case Rep. 2020, 8, 498-502. [CrossRef]

37. Paul, J.-F.; Charles, P.; Richaud, C.; Caussin, C.; Diakov, C. Myocarditis revealing COVID-19 infection in a young patient. Eur. Heart J. Cardiovasc. Imaging 2020, 21, 776. [CrossRef]

38. Pavon, A.G.; Meier, D.; Samim, D.; Rotzinger, D.C.; Fournier, S.; Marquis, P.; Monney, P.; Muller, O.; Schwitter, J. First Documentation of Persistent SARS-Cov-2 Infection Presenting With Late Acute Severe Myocarditis. Can. J. Cardiol. 2020, 36, 1326.e5-1326.e7. [CrossRef] [PubMed]

39. Radbel, J.; Narayanan, N.; Bhatt, P.J. Use of Tocilizumab for COVID-19-Induced Cytokine Release Syndrome. Chest 2020, 158, e15-e19. [CrossRef] [PubMed]

40. Rauf, A.; Vijayan, A.; John, S.T.; Krishnan, R.; Latheef, A. Multisystem Inflammatory Syndrome with Features of Atypical Kawasaki Disease during COVID-19 Pandemic. Indian J. Pediatr. 2020, 87, 745-747. [CrossRef] 
41. Sala, S.; Peretto, G.; Gramegna, M.; Palmisano, A.; Villatore, A.; Vignale, D. Acute myocarditis presenting as a reverse Tako-Tsubo syndrome in a patient with SARS-CoV-2 respiratory infection. Eur. Heart J. 2020, 41, 1861-1862. [CrossRef]

42. Sardari, A.; Tabarsi, P.; Borhany, H.; Mohiaddin, R.; Houshmand, G. Myocarditis detected after COVID-19 recovery. Eur. Heart J. Cardiovasc. Imaging 2021, 22, 131-132. [CrossRef]

43. Tavazzi, G.; Pellegrini, C.; Maurelli, M.; Belliato, M.; Sciutti, F.; Bottazzi, A.; Sepe, P.A.; Resasco, T.; Camporotondo, R.; Bruno, R.; et al. Myocardial localization of coronavirus in COVID-19 cardiogenic shock. Eur. J. Heart Fail. 2020, 22, 911-915. [CrossRef]

44. Trogen, B.; Gonzalez, F.J.; Shust, G.F. COVID-19-Associated Myocarditis in an Adolescent. Pediatr. Infect. Dis. J. 2020, 39 , e204-e205. [CrossRef] [PubMed]

45. Warchoł, I.; Dębska-Kozłowska, A.; Karcz-Socha, I.; Książczyk, M.; Szymańska, K.; Lubiński, A. Terra incognita: Clinically suspected myocarditis in a patient with severe acute respiratory syndrome coronavirus 2 infection. Pol. Arch. Intern. Med. 2020. [CrossRef]

46. Yuan, W.; Tang, X.; Zhao, X. An 'asymptomatic' driver with COVID-19: Atypical suspected myocarditis by SARS-CoV-2. Cardiovasc. Diagn. Ther. 2020, 10, 242-243. [CrossRef]

47. Zeng, J.-H.; Liu, Y.-X.; Yuan, J.; Wang, F.-X.; Wu, W.-B.; Li, J.-X.; Wang, L.-F.; Gao, H.; Wang, Y.; Dong, C.-F.; et al. First case of COVID-19 complicated with fulminant myocarditis: A case report and insights. Infection 2020, 48, 773-777. [CrossRef]

48. Beaudry, J.T.; Dietrick, B.; Lammert, D.B.; Constas, A.; McCaw, J.; Hammond, J.; Buendia, M.; Stein, J.E.; Pekosz, A.; Schuette, J.; et al. Fatal SARS-CoV-2 Inflammatory Syndrome and Myocarditis in an Adolescent: A Case Report. Pediatr. Infect. Dis. J. 2021, 40, e72-e76. [CrossRef]

49. Cairns, L.; El Khaleq, Y.A.; Storrar, W.; Scheuermann-Freestone, M. COVID-19 myopericarditis with cardiac tamponade in the absence of respiratory symptoms: A case report. J. Med. Case Rep. 2021, 15, 31. [CrossRef] [PubMed]

50. Garot, J.; Amour, J.; Pezel, T.; Dermoch, F.; Messadaa, K.; Felten, M.-L.; Raymond, V.; Baubillier, E.; Sanguineti, F.; Garot, P. SARS-CoV-2 Fulminant Myocarditis. JACC Case Rep. 2020, 2, 1342-1346. [CrossRef] [PubMed]

51. Buja, L.M.; Wolf, D.A.; Zhao, B.; Akkanti, B.; McDonald, M.; Lelenwa, L.; Reilly, N.; Ottaviani, G.; Elghetany, M.T.; Trujillo, D.O.; et al. The emerging spectrum of cardiopulmonary pathology of the coronavirus disease 2019 (COVID-19): Report of 3 autopsies from Houston, Texas, and review of autopsy findings from other United States cities. Cardiovasc. Pathol. 2020, 48, 107233. [CrossRef] [PubMed]

52. Chen, C.; Zhou, Y.; Wang, D.W. SARS-CoV-2: A potential novel etiology of fulminant myocarditis. Herz 2020, 45, $230-232$. [CrossRef] [PubMed]

53. Deng, Y.; Liu, W.; Liu, K.; Fang, Y.-Y.; Shang, J.; Zhou, L.; Wang, K.; Leng, F.; Wei, P.-F.; Chen, L.; et al. Clinical characteristics of fatal and recovered cases of coronavirus disease 2019 in Wuhan, China: A retrospective study. Chin. Med. J. 2020, 133, $1261-1267$. [CrossRef] [PubMed]

54. Escher, F.; Pietsch, H.; Aleshcheva, G.; Bock, T.; Baumeier, C.; Elsaesser, A.; Wenzel, P.; Hamm, C.; Westenfeld, R.; Schultheiss, M.; et al. Detection of viral SARS-CoV-2 genomes and histopathological changes in endomyocardial biopsies. ESC Heart Fail. 2020, 7, 2440-2447. [CrossRef] [PubMed]

55. Guo, T.; Fan, Y.; Chen, M.; Wu, X.; Zhang, L.; He, T.; Wang, H.; Wan, J.; Wang, X.; Lu, Z. Cardiovascular Implications of Fatal Outcomes of Patients With Coronavirus Disease 2019 (COVID-19). JAMA Cardiol. 2020, 5, 811-818. [CrossRef] [PubMed]

56. Halushka, M.K.; Heide, R.S.V. Myocarditis is rare in COVID-19 autopsies: Cardiovascular findings across 277 postmortem examinations. Cardiovasc. Pathol. 2021, 50, 107300. [CrossRef]

57. Han, H.; Xie, L.; Liu, R.; Yang, J.; Liu, F.; Wu, K.; Chen, L.; Hou, W.; Feng, Y.; Zhu, C. Analysis of heart injury laboratory parameters in 273 COVID-19 patients in one hospital in Wuhan, China. J. Med. Virol. 2020, 92, 819-823. [CrossRef]

58. Sawalha, K.; Abozenah, M.; Kadado, A.J.; Battisha, A.; Al-Akchar, M.; Salerno, C.; Hernandez-Montfort, J.; Islam, A.M. Systematic Review of COVID-19 Related Myocarditis: Insights on Management and Outcome. Cardiovasc. Revasc. Med. 2021, $23,107-113$. [CrossRef]

59. Duarte-Neto, A.N.; Monteiro, R.A.D.A.; Da Silva, L.F.F.; Malheiros, D.M.A.C.; De Oliveira, E.P.; Theodoro-Filho, J.; Pinho, J.R.R.; Gomes-Gouvêa, M.S.; Salles, A.P.M.; De Oliveira, I.R.S.; et al. Pulmonary and systemic involvement in COVID-19 patients assessed with ultrasound-guided minimally invasive autopsy. Histopathology 2020, 77, 186-197. [CrossRef]

60. Puntmann, V.O.; Carerj, M.L.; Wieters, I.; Fahim, M.; Arendt, C.; Hoffmann, J.; Shchendrygina, A.; Escher, F.; Vasa-Nicotera, M.; Zeiher, A.M.; et al. Outcomes of Cardiovascular Magnetic Resonance Imaging in Patients Recently Recovered From Coronavirus Disease 2019 (COVID-19). JAMA Cardiol. 2020, 5, 1265. [CrossRef]

61. Rajpal, S.; Tong, M.S.; Borchers, J.; Zareba, K.M.; Obarski, T.P.; Simonetti, O.P.; Daniels, C.J. Cardiovascular Magnetic Resonance Findings in Competitive Athletes Recovering From COVID-19 Infection. JAMA Cardiol. 2021, 116-118. [CrossRef] [PubMed]

62. Ruan, Q.; Yang, K.; Wang, W.; Jiang, L.; Song, J. Clinical predictors of mortality due to COVID-19 based on an analysis of data of 150 patients from Wuhan, China. Intensive Care Med. 2020, 46, 846-848. [CrossRef] [PubMed]

63. Shi, S.; Qin, M.; Shen, B.; Cai, Y.; Liu, T.; Yang, F.; Gong, W.; Liu, X.; Liang, J.; Zhao, Q.; et al. Association of cardiac injury with mortality in hospitalized patients with COVID-19 in Wuhan, China. JAMA Cardiol. 2020, 5, 802-810. [CrossRef] [PubMed]

64. Yang, J.; Hu, J.; Zhu, C. Obesity aggravates COVID-19: A systematic review and meta-analysis. J. Med. Virol. 2021, 93, 257-261. [CrossRef] 
65. Azarkish, M.; Laleh Far, V.; Eslami, M.; Mollazadeh, R. Transient complete heart block in a patient with critical COVID-19. Eur. Heart J. 2020, 41, 2131. [CrossRef] [PubMed]

66. Baldi, E.; Sechi, G.M.; Mare, C.; Canevari, F.; Brancaglione, A.; Primi, R.; Klersy, C.; Palo, A.; Contri, E.; Ronchi, V.; et al. Out-of-Hospital Cardiac Arrest during the Covid-19 Outbreak in Italy. N. Engl. J. Med. 2020, 383, 496-498. [CrossRef]

67. Santacroce, L.; D'agostino, D.; Charitos, I.A.; Bottalico, L.; Ballini, A. A short review about electrophysiology and bi-oimpedance: History and perspectives. Indian J. Public Health Res. Dev. 2018, 9, 577-591. [CrossRef]

68. Bhatla, A.; Mayer, M.M.; Adusumalli, S.; Hyman, M.C.; Oh, E.; Tierney, A.; Moss, J.; Chahal, A.A.; Anesi, G.; Denduluri, S.; et al. COVID-19 and cardiac arrhythmias. Hear. Rhythm. 2020, 17, 1439-1444. [CrossRef] [PubMed]

69. Jaffe, A.S.; Cleland, J.G.F.; Katus, H.A. Myocardial injury in severe COVID-19 infection. Eur. Heart J. 2020, 41, $2080-2082$. [CrossRef]

70. Albert, C.L.; Carmona-Rubio, A.E.; Weiss, A.J.; Procop, G.G.; Starling, R.C.; Rodriguez, E.R. The Enemy Within: Sudden-Onset Reversible Cardiogenic Shock With Biopsy-Proven Cardiac Myocyte Infection by Severe Acute Respiratory Syndrome Coronavirus 2. Circulation 2020, 142, 1865-1870. [CrossRef]

71. Ranard, L.S.; Fried, J.A.; Abdalla, M.; Anstey, D.E.; Givens, R.C.; Kumaraiah, D.; Kodali, S.K.; Takeda, K.; Karmpaliotis, D.; Rabbani, L.E.; et al. Approach to Acute Cardiovascular Complications in COVID-19 Infection. Circ Heart Fail. 2020, 13, e007220. [CrossRef]

72. Nicin, L.; Abplanalp, W.T.; Mellentin, H.; Kattih, B.; Tombor, L.; John, D.; Schmitto, J.D.; Heineke, J.; Emrich, F.; Arsalan, M.; et al. Cell type-specific expression of the putative SARS-CoV-2 receptor ACE2 in human hearts. Eur. Heart J. 2020, 41, 1804-1806. [CrossRef] [PubMed]

73. Guan, W.J.; Ni, Z.Y.; Hu, Y.; Liang, W.H.; Ou, C.Q.; He, J.X.; Liu, L.; Shan, H.; Lei, C.L.; Hui, D.S.C.; et al. Clinical Characteristics of Coronavirus Disease 2019 in China. N. Engl. J. Med. 2020, 382, 1708-1720. [CrossRef]

74. Clerkin, K.J.; Fried, J.A.; Raikhelkar, J.; Sayer, G.; Griffin, J.M.; Masoumi, A. COVID-19 and cardiovascular disease. Circulation 2020, 141, 1648-1655. [CrossRef] [PubMed]

75. Colon, C.M.; Barrios, J.G.; Chiles, J.W.; McElwee, S.K.; Russell, D.W.; Maddox, W.R.; Kay, G.N. Atrial Arrhythmias in COVID-19 Patients. JACC Clin. Electrophysiol. 2020, 6, 1189-1190. [CrossRef] [PubMed]

76. Fox, S.E.; Akmatbekov, A.; Harbert, J.L.; Li, G.; Brown, J.Q.; Heide, R.S.V. Pulmonary and cardiac pathology in African American patients with COVID-19: An autopsy series from New Orleans. Lancet Respir. Med. 2020, 8, 681-686. [CrossRef]

77. Lindner, D.; Fitzek, A.; Bräuninger, H.; Aleshcheva, G.; Edler, C.; Meissner, K.; Scherschel, K.; Kirchhof, P.; Escher, F.; Schultheiss, H.-P.; et al. Association of Cardiac Infection With SARS-CoV-2 in Confirmed COVID-19 Autopsy Cases. JAMA Cardiol. 2020, 5, 1281. [CrossRef] [PubMed]

78. McMurray, J.C.; May, J.W.; Cunningham, M.W.; Jones, O.Y. Multisystem Inflammatory Syndrome in Children (MIS-C), a Postviral Myocarditis and Systemic Vasculitis-A Critical Review of Its Pathogenesis and Treatment. Front. Pediatr. 2020, 8, 626182. [CrossRef]

79. Babapoor-Farrokhran, S.; Rasekhi, R.T.; Gill, D.; Babapoor, S.; Amanullah, A. Arrhythmia in COVID-19. SN Compr. Clin. Med. 2020, 2, 1430-1435. [CrossRef]

80. Lazzerini, P.E.; Boutjdir, M.; Capecchi, P.L. COVID-19, Arrhythmic Risk, and Inflammation: Mind the Gap! Circulation 2020, 142, 7-9. [CrossRef] [PubMed]

81. Buja, L.M.; Stone, J.R. A novel coronavirus meets the cardiovascular system: Society for Cardiovascular Pathology Symposium 2021. Cardiovasc. Pathol. 2021, 53, 107336. [CrossRef] [PubMed]

82. Barber, C. COVID-19 Can Wreck Your Heart, Even if You Haven't Had Any Symptoms. Sci. Am. 2020. Available online: https: //www.scientificamerican.com/article/covid-19-can-wreck-your-heart-even-if-you-havent-had-any-symptoms / (accessed on 15 May 2021).

83. Santacroce, L.; Charitos, I.A.; Ballini, A.; Inchingolo, F.; Luperto, P.; De Nitto, E.; Topi, S. The Human Respiratory System and its Microbiome at a Glimpse. Biology 2020, 9, 318. [CrossRef] [PubMed]

84. Djomkam, A.L.Z.; Olwal, C.O.; Sala, T.B.; Paemka, L. Commentary: SARS-CoV-2 Cell Entry Depends on ACE2 and TMPRSS2 and Is Blocked by a Clinically Proven Protease Inhibitor. Front. Oncol. 2020, 10, 1448. [CrossRef] [PubMed]

85. Blauwet, L.A.; Cooper, L.T. Myocarditis. Prog. Cardiovasc. Dis. 2010, 52, 274-288. [CrossRef]

86. Rubini, G.; Ferrari, C.; Carretta, D.; Santacroce, L.; Ruta, R.; Iuele, F.; Lavelli, V.; Merenda, N.; D’Agostino, C.; Sardaro, A.; et al. Usefulness of ${ }^{18}$ F-FDG PET/CT in Patients with Cardiac Implantable Electronic Device Suspected of Late Infection. J. Clin. Med. 2020, 9, 2246. [CrossRef] [PubMed]

87. Birchak, J.; Khan, A.; Singh, G.; Schuger, C.; Maskoun, W. An unusual case of sustained ventricular tachycardia from acute pulmonary embolism. J. Am. Coll. Cardiol. 2020, 75, 2820. [CrossRef]

88. Biesbroek, P.S.; Beek, A.M.; Germans, T.; Niessen, H.W.; van Rossum, A.C. Diagnosis of myocarditis: Current state and future perspectives. Int. J. Cardiol. 2015, 191, 211-219. [CrossRef] [PubMed]

89. Channappanavar, R.; Zhao, J.; Perlman, S. T cell-mediated immune response to respiratory coronaviruses. Immunol. Res. 2014, 59, 118-128. [CrossRef] [PubMed]

90. Gnoni, A.; Ballini, A.; Trentadue, R.; Taurino, F.; Santacroce, L.; Ferrara, P.; Massaro, F.; Brienza, N.; Massari, A.M.; Sardaro, N.; et al. Induction of mitochondrial dysfunction in patients under cardiopulmonary by-pass: Preliminary results. Eur. Rev. Med. Pharmacol. Sci. 2019, 23, 8115-8123. 
91. Caforio, A.L.P.; Pankuweit, S.; Arbustini, E.; Basso, C.; Gimeno-Blanes, J.; Felix, S.B.; Fu, M.; Heliö, T.; Heymans, S.; Jahns, R.; et al. Current state of knowledge on aetiology, diagnosis, management, and therapy of myocarditis: A position statement of the European Society of Cardiology Working Group on Myocardial and Pericardial Diseases. Eur. Heart J. 2013, 34, $2636-2648$. [CrossRef]

92. Yang, C.; Jin, Z. An Acute Respiratory Infection Runs Into the Most Common Noncommunicable Epidemic-COVID-19 and Cardiovascular Diseases. JAMA Cardiol. 2020, 5, 743-744. [CrossRef]

93. Roshdy, A.; Zaher, S.; Fayed, H.; Coghlan, J.G. COVID-19 and the Heart: A Systematic Review of Cardiac Autopsies. Front Cardiovasc. Med. 2021, 7, 626975. [CrossRef]

94. Goyal, P.; Choi, J.J.; Pinheiro, L.C.; Schenck, E.J.; Chen, R.; Jabri, A.; Satlin, M.J.; Campion, T.R.; Nahid, M.; Ringel, J.B.; et al. Clinical Characteristics of Covid-19 in New York City. N. Engl. J. Med. 2020, 382, 2372-2374. [CrossRef]

95. Dabbagh, M.F.; Aurora, L.; D'Souza, P.; Weinmann, A.J.; Bhargava, P.; Basir, M.B. Cardiac Tamponade Secondary to COVID-JACC. Case Rep. 2020, 2, 1326-1330. [CrossRef]

96. Grasselli, G.; Zangrillo, A.; Zanella, A.; Antonelli, M.; Cabrini, L.; Castelli, A.; Cereda, D.; Coluccello, A.; Foti, G.; Fumagalli, R.; et al. Baseline Characteristics and Outcomes of 1591 Patients Infected with SARS-CoV-2 Admitted to ICUs of the Lombardy Region, Italy. JAMA 2020, 323, 1574-1581. [CrossRef]

97. Thakkar, S.; Arora, S.; Kumar, A.; Jaswaney, R.; Faisaluddin, M.; Ammad Ud Din, M.; Shariff, M.; Barssoum, K.; Patel, H.P.; Nirav, A.; et al. A Systematic Review of the Cardiovascular Manifestations and Outcomes in the Setting of Coronavirus-19 Disease. Clin. Med. Insights Cardiol. 2020, 14, 1179546820977196. [CrossRef] [PubMed]

98. Lakkireddy, D.R.; Chung, M.K.; Gopinathannair, R.; Patton, K.K.; Gluckman, T.J.; Turagam, M.; Cheung, J.W.; Patel, P.; Sotomonte, J.; Lampert, R.; et al. Guidance for cardiac electrophysiology during the COVID-19 pandemic from the Heart Rhythm Society COVID-19 Task Force; Electrophysiology Section of the American College of Cardiology; and the Electrocardiography and Arrhythmias Committee of the Council on Clinical Cardiology, American Heart Association. Heart Rhythm. 2020, 17, e233-e241. [CrossRef] [PubMed]

99. Nieminen, M.S.; Heikkilä, J.; Karjalainen, J. Echocardiography in acute infectious myocarditis: Relation to clinical and electrocardiographic findings. Am. J. Cardiol. 1984, 53, 1331-1337. [CrossRef]

100. Felker, G.M.; Boehmer, J.P.; Hruban, R.H.; Hutchins, G.M.; Kasper, E.K.; Baughman, K.L. Echocardiographic findings in fulminant and acute myocarditis. J. Am. Coll. Cardiol. 2000, 36, 227-232. [CrossRef]

101. Friedrich, M.G.; Sechtem, U.; Schulz-Menger, J.; Holmvang, G.; Alakija, P.; Cooper, L.T.; White, J.A.; Abdel-Aty, H.; Gutberlet, M.; Prasad, S.; et al. Cardiovascular Magnetic Resonance in Myocarditis: A JACC White Paper. J. Am. Coll. Cardiol. 2009, 53, 1475-1487. [CrossRef] [PubMed]

102. Fung, G.; Luo, H.; Qiu, Y.; Yang, D.; McManus, B. Myocarditis. Circ. Res. 2016, 118, 496-514. [CrossRef]

103. Chen, H.S.; Wang, W.; Wu, S.N.; Liu, J.P. Corticosteroids for viral myocarditis. Cochrane Database Syst. Rev. 2013, 2013, CD004471. [CrossRef] [PubMed]

104. Verhoef, P.A.; Kannan, S.; Sturgill, J.L.; Tucker, E.W.; Morris, P.E.; Miller, A.C.; Sexton, T.R.; Koyner, J.L.; Hejal, R.; Brakenridge, S.C.; et al. Severe Acute Respiratory Syndrome-Associated Coronavirus 2 Infection and Organ Dysfunction in the ICU: Opportunities for Translational Research. Crit. Care Explor. 2021, 3, e0374. [CrossRef]

105. Wu, C.; Chen, X.; Cai, Y.; Xia, J.; Zhou, X.; Xu, S.; Huang, H.; Zhang, L.; Zhou, X.; Du, C.; et al. Risk Factors Associated with Acute Respiratory Distress Syndrome and Death in Patients With Coronavirus Disease 2019 Pneumonia in Wuhan, China. JAMA Intern. Med. 2020, 180, 934-943. [CrossRef]

106. Hasan, S.S.; Capstick, T.; Ahmed, R.; Kow, C.S.; Mazhar, F.; Merchant, H.A.; Zaidi, S.T.R. Mortality in COVID-19 patients with acute respiratory distress syndrome and corticosteroids use: A systematic review and meta-analysis. Expert Rev. Respir. Med. 2020, 14, 1149-1163. [CrossRef]

107. Lythgoe, M.P.; Middleton, P. Ongoing Clinical Trials for the Management of the COVID-19 Pandemic. Trends Pharmacol. Sci. 2020, 41, 363-382. [CrossRef]

108. Kow, C.S.; Hasan, S.S. Glucocorticoid versus immunoglobulin in the treatment of COVID-19-associated fulminant myocarditis. Infection 2020, 48, 805-806. [CrossRef] [PubMed]

109. Li, Y.; Yu, Y.; Chen, S.; Liao, Y.; Du, J. Corticosteroids and Intravenous Immunoglobulin in Pediatric Myocarditis: A Meta-Analysis. Front. Pediatr. 2019, 7, 342. [CrossRef]

110. Jin, Y.-H.; Cai, L.; Cheng, Z.-S.; Cheng, H.; Deng, T.; Fan, Y.-P.; Fang, C.; Huang, D.; Huang, L.-Q.; Huang, Q.; et al. A rapid advice guideline for the diagnosis and treatment of 2019 novel coronavirus (2019-nCoV) infected pneumonia (standard version). Mil. Med. Res. 2020, 7, 4. [CrossRef] [PubMed]

111. Farooqi, F.; Dhawan, N.; Morgan, R.; Dinh, J.; Nedd, K.; Yatzkan, G. Treatment of Severe COVID-19 with Tocilizumab Mitigates Cytokine Storm and Averts Mechanical Ventilation During Acute Respiratory Distress: A Case Report and Literature Review. Trop. Med. Infect. Dis. 2020, 5, 112. [CrossRef] [PubMed]

112. Beigel, J.H.; Tomashek, K.M.; Dodd, L.E.; Mehta, A.K.; Zingman, B.S.; Kalil, A.C.; Hohmann, E.; Chu, H.Y.; Luetkemeyer, A.; Kline, S.; et al. Remdesivir for the Treatment of Covid-19-Final Report. N. Engl. J. Med. 2020, 383, 1813-1826. [CrossRef]

113. Castiglione, V.; Chiriacò, M.; Emdin, M.; Taddei, S.; Vergaro, G. Statin therapy in COVID-19 infection. Eur. Heart J. Cardiovasc. Pharmacol. 2020, 6, 258-259. [CrossRef] 
114. European Society of Cardiology Working Group on Myocardial and Pericardial Diseases guidelines. Available online: https: / / www.escardio.org/Guidelines (accessed on 1 December 2020).

115. Fiore, A.; Piscitelli, M.; Adodo, D.K.; Thomas, C.; Dessap, A.M.; Bagate, F.; Folliguet, T. Successful Use of Extracorporeal Membrane Oxygenation Postpartum as Rescue Therapy in a Woman With COVID-19. J. Cardiothorac. Vasc. Anesth. 2020, 35, 2140-2143. [CrossRef] [PubMed]

116. Alom, S.; Haiduc, A.A.; Melamed, N.; Axiaq, A.; Harky, A. Use of ECMO in Patients With Coronavirus Disease 2019: Does the Evidence Suffice? J. Cardiothorac. Vasc. Anesth. 2021, 35, 1256-1262. [CrossRef]

117. Charitos, I.A.; Topi, S.; Castellaneta, F.; D'Agostino, D. Current Issues and Perspectives in Patients with Possible Sepsis at Emergency Departments. Antibiotics 2019, 8, 56. [CrossRef]

118. Siripanthong, B.; Nazarian, S.; Muser, D.; Deo, R.; Santangeli, P.; Khanji, M.Y.; Cooper, L.T.; Chahal, C.A.A. Recognizing COVID19-related myocarditis: The possible pathophysiology and proposed guideline for diagnosis and management. Heart Rhythm. 2020, 17, 1463-1471. [CrossRef]

119. Topol, E.J. COVID-19 can affect the heart. Science 2020, 370, 408-409. [CrossRef]

120. Bonow, R.O.; Yancy, C.W. Explanation for the Corrections for the Study of Cardiovascular Magnetic Resonance Imaging in Patients Recently Recovered From Coronavirus Disease 2019. JAMA Cardiol. 2020, 5, 1308. [CrossRef]

121. Ho, J.S.; Sia, C.H.; Chan, M.Y.; Lin, W.; Wong, R.C. Coronavirus-induced myocarditis: A meta-summary of cases. Heart Lung 2020, 49, 681-685. [CrossRef]

122. Salman, D.; Vishnubala, D.; Le Feuvre, P.; Beaney, T.; Korgaonkar, J.; Majeed, A.; McGregor, A.H. Returning to physical activity after covid-19. BMJ 2021, 372, m4721. [CrossRef]

123. Centers for Disease Control and Prevention (CDC), USA. Post-COVID 19 Conditions. 2021. Available online: https://www.cdc. gov/coronavirus /2019-ncov/long-term-effects.html (accessed on 20 May 2021). 